\title{
Analysis of a quantum subband model for the transport of partially confined charged particles
}

\author{
Florian Méhats \\ MIP, Laboratoire CNRS (UMR 5640), Université Paul Sabatier, \\ 118, route de Narbonne, 31062 Toulouse Cedex 04, France \\ mehats@mip.ups-tlse.fr
}

\begin{abstract}
This paper is devoted to the analysis of a quantum subband model, which is presented as an alternative to the standard 3D Schrödinger-Poisson system for modeling the transport of electrons strongly confined along one direction. This subband model is composed of quasistatic 1D Schrödinger equations in the direction of the confinement, coupled to 2D time-dependent Schrödinger equations describing the transport in the non-confined directions. Selfconsistent electrostatic interactions are also taken into account via the Poisson equation. This system is studied in the framework of the strong partial confinement asymptotics introduced in the article "Adiabatic approximation of the Schrödinger-Poisson system with a partial confinement", by Ben Abdallah, Méhats and Pinaud (to appear in SIAM J. Math. Anal.).
\end{abstract}

\section{Introduction}

As electronic devices shrink to nanometric scales, the numerical simulation of their operation requires multidimensional quantum transport models in the ballistic regime. In many nanoscale semiconductor devices like ultrashort channel doublegate MOSFETs, electrons might be extremely confined in one direction transverse to the transport directions. The mode-space approach, also referred to as subband decomposition method [30,31, 9, 10,32], was recently introduced by several authors in order to take advantage of this reduction of dimensionality and design efficient numerical codes. This method consists of a "block diagonalisation" of the electron Hamiltonian thanks to a separation of the confinement and transport directions. The computational gain is significant, the 3D Schrödinger equation being replaced by $1 \mathrm{D}$ Schrödinger equations (for the confinement) coupled to $2 \mathrm{D}$ equations (for the transport).

The aim of this paper is to analyze mathematically a quantum subband model, including selfconsistent Coulombian interactions, and to appreciate the relevance of the approximations made in this procedure of separation of variables. This work is put in perspective with [6], where an asymptotics for strong confinement was used to derive a hierarchy of quantum transport models for bidimensional electron gases (see also [25] concerning the stationary case). 
Thus, our starting model is the same as in [6]. We consider the 3D SchrödingerPoisson system with a strong applied partial confinement potential. Denote by $z \in \mathbb{R}$ the confinement (or transverse) direction and by $x \in \mathbb{R}^{2}$ the transport directions. The initial 3D system is written:

$$
\begin{gathered}
i \partial_{t} \psi^{3 \mathrm{D}}=-\frac{1}{2} \Delta_{x, z} \psi^{3 \mathrm{D}}+\left(V_{c}^{\varepsilon}+V^{3 \mathrm{D}}\right) \psi^{3 \mathrm{D}}, \\
V^{3 \mathrm{D}}=\frac{1}{4 \pi r} *\left(\left|\psi^{3 \mathrm{D}}\right|^{2}\right),
\end{gathered}
$$

where $r=\sqrt{|x|^{2}+z^{2}}$. The unknowns are the wavefunction $\psi^{3 \mathrm{D}}$ and the selfconsistent potential $V^{3 \mathrm{D}}$. In this system, $\varepsilon$ is a dimensionless small parameter and $V_{c}^{\varepsilon}$ denotes the applied confinement potential, depending only on the $z$ variable, and which is obtained after the following rescaling of a function $V_{c}$ independent of $\varepsilon$ :

$$
V_{c}^{\varepsilon}(z)=\frac{1}{\varepsilon^{2}} V_{c}\left(\frac{z}{\varepsilon}\right)
$$

The hypotheses on this function $V_{c}$ are made precise below (see Assumptions 2.2 and 2.3). The parameter $\varepsilon$ is the square root of the ratio between the kinetic energy in the non-confined directions and the confinement energy (see [6]). It is also the order of magnitude of the gas thickness (rescaled by a characteristic length of the transport).

The confinement modes, usually referred to as the subbands of the system in the physics literature $[1,3,14,16]$, are defined as the eigenfunctions of the transverse part of the Hamiltonian. More precisely, the variables $t, x$ being frozen, consider the stationary Schrödinger equation in the variable $z \in \mathbb{R}$ :

$$
-\frac{1}{2} \partial_{z}^{2} \chi_{p}^{3 \mathrm{D}}+\left(V_{c}^{\varepsilon}+V^{3 \mathrm{D}}\right) \chi_{p}^{3 \mathrm{D}}=\epsilon_{p}^{3 \mathrm{D}} \chi_{p}^{3 \mathrm{D}} .
$$

The whole sequence of real-valued eigenfunctions $\left(\chi_{p}^{3 \mathrm{D}}(t, x, z)\right)_{p \in \mathbb{N}^{*}}$, associated to the sequence of increasing eigenvalues $\left(\epsilon_{p}^{3 \mathrm{D}}(t, x)\right)_{p \in \mathbb{N}^{*}}$, defines the subbands of the system and can serve as a basis for evaluating the wavefunction of the 3D system. Equation (1.1) can be equivalently rewritten in this basis, but it is readily seen that, due to the parametric dependence of the $\chi_{p}^{3 \mathrm{D}}$ 's in the variables $t$ and $x$, this leads to an infinite system of coupled 2D Schrödinger equations. The quantum subband model consists in neglecting the coupling terms in this system. In particular, this amounts to consider that the total Hamiltonian $-\frac{1}{2} \Delta_{x}-\frac{1}{2} \partial_{z}^{2}+V_{c}^{\varepsilon}+V^{3 \mathrm{D}}$ is block-diagonal in the basis of the $\chi_{p}^{3 \mathrm{D}}$ 's: it is assumed that within each subband, its action reduces to the action of the operator $-\frac{1}{2} \Delta_{x}+\epsilon_{p}^{3 \mathrm{D}}$. Let us rewrite this approximate system (to avoid confusions, the unknowns of this subband model are now labelled by ${ }^{\mathrm{SB}}$ ). For each $p \in \mathbb{N}^{*}$, we consider the wavefunction $\phi_{p}^{\mathrm{SB}}(t, x)$ solving a time-dependent Schrödinger equation in dimension 2 :

$$
i \partial_{t} \phi_{p}^{\mathrm{SB}}=-\frac{1}{2} \Delta_{x} \phi_{p}^{\mathrm{SB}}+\epsilon_{p}^{\mathrm{SB}} \phi_{p}^{\mathrm{SB}}
$$


Cauchy data are given for this system of decoupled evolution equations: $\phi_{p}^{\text {SB }}(0, x)=$ $\phi_{p, 0}(x)$ for $p \in \mathbb{N}^{*}$. The dynamics of the $p$ th 2D Schrödinger equation is driven by an effective potential $\epsilon_{p}^{\mathrm{SB}}$, which is defined as the $p$ th eigenvalue of the partial Hamiltonian $-\partial_{z}^{2}+V_{c}^{\varepsilon}+V^{\mathrm{SB}}$ :

$$
-\frac{1}{2} \partial_{z}^{2} \chi_{p}^{\mathrm{SB}}+\left(V_{c}^{\varepsilon}+V^{\mathrm{SB}}\right) \chi_{p}^{\mathrm{SB}}=\epsilon_{p}^{\mathrm{SB}} \chi_{p}^{\mathrm{SB}}
$$

The electronic system is described by a mixed quantum state formed of pure states located on the subbands. Its density matrix has the kernel

$$
\varrho\left(t, x, x^{\prime}, z, z^{\prime}\right)=\sum_{p \geq 1} \phi_{p}^{\mathrm{SB}}(t, x) \chi_{p}^{\mathrm{SB}}(t, x, z) \overline{\phi_{p}^{\mathrm{SB}}}\left(t, x^{\prime}\right) \chi_{p}^{\mathrm{SB}}\left(t, x^{\prime}, z^{\prime}\right) .
$$

The electron charge density is thus defined as the diagonal of this density matrix:

$$
n^{\mathrm{SB}}(t, x, z)=\sum_{p \geq 1}\left|\phi_{p}^{\mathrm{SB}}\right|^{2}(t, x)\left|\chi_{p}^{\mathrm{SB}}\right|^{2}(t, x, z) .
$$

In other words, another approximation has been made in the 3D system, consisting in neglecting the phase coherence between the components of the wavefunction on the various subbands. To complete the quantum subband model, it remains to write the Poisson equation satisfied by the selfconsistent potential $V^{\text {sB }}$ :

$$
V^{\mathrm{SB}}=\frac{1}{4 \pi r} *\left(\sum_{p \geq 1}\left|\phi_{p}^{\mathrm{SB}}\right|^{2}\left|\chi_{p}^{\mathrm{SB}}\right|^{2}\right) .
$$

This paper is devoted to the study of this model (1.4), (1.5), (1.6). Two kinds of results are given. Firstly, it is shown that this system is well-posed, at least for $\varepsilon$ small enough: this result is stated in Theorem 2.5. Secondly, this model is compared to two other models: the 3D Schrödinger-Poisson model (1.1), (1.2) and its limit as $\varepsilon \rightarrow 0$, called the $2 D$ surface density model. Theorem 2.7 (together with Remark 2.8) states that, for well-prepared data, the discrepancy between the quantum subband model and the 3D Schrödinger-Poisson model is of order $\mathcal{O}\left(\varepsilon^{3}\right)$, while the limit 2D model is only an approximation of order $\mathcal{O}(\varepsilon)$ of the 3D model.

Let us end this introduction with a few other bibliographical notes. The 3D Schrödinger-Poisson system (or its density-matrix version, the von Neumann-Poisson system) has been solved in $[2,11,19]$ in the framework of energy spaces, and in [12] in the $L^{2}$ framework. Important tools for such systems, which we also use in the asymptotic analysis of Section 4, are Strichartz-type inequalities [18, 28, 34]. One can also refer to the review [13] (and to references therein), where this system is studied among more general nonlinear Schrödinger equations. The stationary Schrödinger-Poisson system was solved in [21, 22, 23] by variational methods. The strong confinement scaling used in (1.1) was also used in [17] for deriving rigourously a model of quantum transport constraint on general surfaces by external forces (in the linear case). In [29], it is remarked that this problem bears strong analogies with the 
Born-Oppenheimer approximation. In some situations, the length and time scales in the non-confined directions $x$ may be such that the transport can be considered as classical. Then a classical model -kinetic or diffusive- can be used with benefit for the transport within each subband in place of the 2D Schrödinger equations (1.4). Such quantum-classical subband models were derived in [5] and studied in $[4,7,8]$.

This paper is organized as follows. In the next section, we introduce some useful notations and state the main results of this paper, Theorems 2.5 and 2.7. These theorems are respectively proved in Sections 3 and 4. In Section 5, we prove an additional asymptotic result. Another approximate model for the 3D SchrödingerPoisson system is presented: the 2.5D adiabatic model, which was introduced and studied in [6]. Here we show that the difference between the quantum subband model and this 2.5D adiabatic model is of order $\mathcal{O}\left(\varepsilon^{3}\right)$. One of the by-products of this asymptotic analysis is then an improvement of estimates that were proved in [6], where the 3D model and the 2.5D adiabatic model were compared (see Remark 5.2). To this viewpoint, the subband decomposition (1.5) can also be seen as a mathematical tool useful to study this strong confinement asymptotics.

\section{Notations and main results}

\subsection{Functional spaces, notations}

The following functional spaces are used in this paper: for any $1 \leq p, q \leq \infty$,

$$
L_{x}^{p} L_{z}^{q}=\left\{u \in L_{l o c}^{1}\left(\mathbb{R}^{3}\right), \quad\|u\|_{p, q}=\left(\int_{\mathbb{R}^{2}}\|u(x, \cdot)\|_{L^{q}(\mathbb{R})}^{p} d x\right)^{1 / p}<+\infty\right\}
$$

(with an obvious generalization of this definition for $p=+\infty$ ). For any Banach space $E$ and $q \geq 1, \ell^{q}(E)$ denotes the space of sequences $\left(h_{p}\right)_{p \in \mathbb{N}^{*}}$ such that for all $p \geq 1$ we have $h_{p} \in E$ and such that $\left(\sum_{p \geq 1}\left\|h_{p}\right\|^{q}\right)^{1 / q}<+\infty$, the last quantity being the norm of $\left(h_{p}\right)_{p \in \mathbb{N}^{*}}$ in $\ell^{q}(E)$.

Throughout this paper, we use the following notations, for any $q \in[2, \infty]$ :

$$
q^{\prime}=\frac{q}{q-1}, \quad q^{*}=\frac{2 q}{q-2}, \quad q^{\#}=\frac{2 q}{q+2} .
$$

Furthermore, $\|\cdot\|_{q}$ denotes the $L^{q}$ norm and the notation $\langle\cdot\rangle$ stands for an integration with respect to the $z$ variable: $\langle u\rangle=\int_{\mathbb{R}} u(z) d z$.

The following Lemma was proved in [6] and deals with the convolution in dimension 3 of the Poisson kernel $\frac{1}{4 \pi r}$ with $L_{x}^{p} L_{z}^{1}$ densities:

Lemma 2.1 Let $q \in(2, \infty)$. (i) For any $f \in L_{x}^{q^{\#}} L_{z}^{1}$, we have

$$
\left\|\frac{1}{r} * f\right\|_{q, \infty}+\left\|\nabla_{x, z}\left(\frac{1}{r} * f\right)\right\|_{q, 1} \leq C_{q}\|f\|_{q^{\#, 1}} .
$$


If in addition $\nabla_{x} f \in L_{x}^{q^{\#}} L_{z}^{1}$ then

$$
\left\|\nabla_{x, z}\left(\frac{1}{r} * f\right)\right\|_{q, \infty} \leq C_{q}\left\|\nabla_{x} f\right\|_{q^{\#, 1}} .
$$

(ii) For any $f \in L_{x}^{q} L_{z}^{1} \cap L^{1}\left(\mathbb{R}^{3}\right)$, we have

$$
\left\|\frac{1}{r} * f\right\|_{L^{\infty}\left(\mathbb{R}^{3}\right)}+\left\|\nabla_{x, z}\left(\frac{1}{r} * f\right)\right\|_{\infty, 1} \leq C_{q}\|f\|_{q, 1}^{\theta}\|f\|_{L^{1}\left(\mathbb{R}^{3}\right)}^{1-\theta},
$$

where $\theta=\frac{q}{2 q-2}$. If in addition $\nabla_{x} f \in L_{x}^{q} L_{z}^{1} \cap L^{1}\left(\mathbb{R}^{3}\right)$ then

$$
\left\|\nabla_{x, z}\left(\frac{1}{r} * f\right)\right\|_{L^{\infty}\left(\mathbb{R}^{3}\right)} \leq C_{q}\left\|\nabla_{x} f\right\|_{q, 1}^{\theta}\left\|\nabla_{x} f\right\|_{L^{1}\left(\mathbb{R}^{3}\right)}^{1-\theta} .
$$

\subsection{Well-posedness of the quantum subband model}

Let us start with the assumptions on the confinement potential.

Assumption 2.2 The external potential $V_{c}=V_{c}(z)$ is a nonnegative function in $L_{l o c}^{2}\left(\mathbb{R}^{2}\right)$ and such that $V_{c}(z) \rightarrow+\infty$ as $|z| \rightarrow+\infty$.

Thanks to this assumption, one can show that the Hamiltonian $-\frac{1}{2} \frac{d^{2}}{d z^{2}}+V_{c}$ on $L^{2}(\mathbb{R})$ with the domain

$$
\left\{\psi \in H^{2}(\mathbb{R}): \quad V_{c} \psi \in L^{2}(\mathbb{R})\right\}
$$

admits a discrete simple spectrum and a complete set of eigenfunctions (see for instance [26]). Its eigenvalues and eigenfunctions are denoted by $E_{p}$ and $X_{p}$. With the notation $V_{c}^{\varepsilon}(z)=\frac{1}{\varepsilon^{2}} V_{c}\left(\frac{z}{\varepsilon}\right)$, the spectral elements of the Hamiltonian $-\frac{1}{2} \frac{d^{2}}{d z^{2}}+V_{c}^{\varepsilon}$ are obtained by a simple rescaling:

$$
\frac{E_{p}}{\varepsilon^{2}}, \quad X_{p}^{\varepsilon}(z)=\frac{1}{\sqrt{\varepsilon}} X_{p}\left(\frac{z}{\varepsilon}\right) .
$$

Additionnaly, we assume that the confinement potential is such that

Assumption 2.3 The eigenvalues of $-\frac{1}{2} \frac{d^{2}}{d z^{2}}+V_{c}$ satisfy

$$
\delta_{0}:=\min _{\substack{p, k \in \mathbb{N}^{*} \\ k \neq p}}\left|E_{p}-E_{k}\right|>0 .
$$

Harmonic potentials $V_{c}(z)=\kappa z^{2}$ are simple cases where this Assumption 2.3 is fulfilled. It is also satisfied for superharmonic potentials such that $V_{c}(z) \geq C|z|^{\beta}$ with $\beta>2$, since then a bound from below $E_{p} \geq C p^{2 \beta /(2+\beta)}$ can be deduced from the asymptotic theory of eigenvalues for one-dimensional Schrödinger operators (see for instance [15]). 
A direct consequence of this Assumption 2.3 is that for all $p, k$ we have $\left|E_{p}-E_{k}\right|>$ $\delta_{0}|p-k|$, thus there exists a constant $C_{\alpha}$, independent on $p$, such that

$$
\forall \alpha>1, \quad \forall p \in \mathbb{N}^{*}, \quad \sum_{k \neq p} \frac{1}{\left(E_{p}-E_{k}\right)^{\alpha}}<\frac{C_{\alpha}}{\delta_{0}^{\alpha}} .
$$

Our first theorem states that, at least for small $\varepsilon$ 's, the quantum subband system is well-posed in an energy space, i.e. for initial data satisfying:

Assumption 2.4 The Cauchy data $\phi_{p, 0}$ for (1.4) belong to $H^{1}\left(\mathbb{R}^{2}\right)$ and are such that

$$
\sum_{p \geq 1}\left\|\nabla_{x} \phi_{p, 0}\right\|_{2}^{2}+\sum_{p \geq 1} E_{p}\left\|\phi_{p, 0}\right\|_{2}^{2}<+\infty .
$$

Theorem 2.5 Let $M>0$ and $q \in(2, \infty)$. Then there exists $\varepsilon(M, q)>0$ such that, under Assumption 2.2, 2.3, 2.4 and if

$$
0<\varepsilon<\varepsilon(M, q) \quad ; \quad \sum_{p \geq 1}\left\|\phi_{p, 0}\right\|_{H^{1}}^{2}<M,
$$

the system (1.4), (1.5), (1.6) admits a unique global solution with the properties

$$
\begin{gathered}
\left(\phi_{p}^{\mathrm{SB}}\right)_{p \geq 1} \in C\left([0,+\infty), \ell^{2}\left(H^{1}\left(\mathbb{R}^{2}\right)\right)\right), \\
V^{\mathrm{SB}} \in C\left([0,+\infty), L^{\infty}\left(\mathbb{R}^{3}\right) \cap L_{x}^{q} L_{z}^{\infty}\right), \quad \nabla_{x, z} V^{\mathrm{SB}} \in C\left([0,+\infty), L^{2}\left(\mathbb{R}^{3}\right) \cap L_{x}^{q} L_{z}^{\infty}\right), \\
\forall p \in \mathbb{N}^{*} \quad \epsilon_{p}^{\mathrm{SB}}-\frac{E_{p}}{\varepsilon^{2}} \in C\left([0,+\infty), W^{1, q}\left(\mathbb{R}^{2}\right)\right), \\
\forall p \in \mathbb{N}^{*} \quad \chi_{p}^{\mathrm{SB}}-X_{p}^{\varepsilon} \in C\left([0,+\infty), L_{x}^{q} L_{z}^{2}\right), \quad \nabla_{x} \chi_{p}^{\mathrm{SB}} \in C\left([0,+\infty), L_{x}^{q} L_{z}^{2}\right) .
\end{gathered}
$$

These functions are uniformly bounded in these spaces with respect to $p$ and to the variable $\varepsilon \in(0, \min (1, \varepsilon(M, q)))$. Moreover, if the initial data are such that $\phi_{p, 0} \equiv 0$ for all $p \geq 2$, one can choose $\varepsilon(M, q)=+\infty$.

The last statement of the theorem says that the system always admits a unique solution (even for large $\varepsilon$ 's) in the special case where only the first subband is occupied. This situation is usually referred to as the electrical quantum limit $[3,14,16]$. In the general case, we can only solve this system for small $\varepsilon$ 's and the question of its well-posedness for a large $\varepsilon$ is open. One can explain this restriction as follows. Our strategy of proof consists in reinterpreting the system as a series of time-dependent Schrödinger equations (1.4) in which the potential terms $\epsilon_{p}^{\varepsilon}$ are calculated selfconsistently by solving quasistatic Schrödinger-Poisson systems (1.5), (1.6). With this method, it is crucial to show that these quasistatic Schrödinger-Poisson systems can be solved continuously with respect to their parameters. However, in Subsection 3.1, we are only able to solve them for small $\varepsilon$ 's: this is the reason of the limitation on $\varepsilon$ in Theorem 2.5. A similar problem was encountered in [4], where a quantum-kinetic subband model was studied. 


\subsection{Asymptotic analysis}

The second main result of this paper deals with the asymptotics $\varepsilon \rightarrow 0$. We justify the statement made in the Introduction, where the quantum subband model was presented as an approximation of the 3D Schrödinger-Poisson system. Error estimates between these models are obtained on a time interval $[0, T], T$ being arbitrarily fixed. We shall denote shortly any space $L^{\alpha}\left((0, T), L^{\beta}\left(\mathbb{R}^{2}, L^{\gamma}(\mathbb{R})\right)\right)$ by $L_{t}^{\alpha} L_{x}^{\beta} L_{z}^{\gamma}$. For this asymptotic analysis, a particular class of initial data is considered: we consider subband models where only one subband (labelled by $p$, the integer $p$ being fixed) is populated. Assumption 2.4 is replaced by

Assumption 2.6 There exists $p \in \mathbb{N}^{*}$ such that, initially, only the pth subband is occupied:

- the Cauchy data for the quantum subband model (1.4), (1.5), (1.6) are such that

$$
\phi_{p, 0} \in H^{3}\left(\mathbb{R}^{2}\right) \quad \text { and } \quad \phi_{q, 0} \equiv 0 \quad \text { for any } q \in \mathbb{N}^{*} \backslash\{p\}
$$

- the Cauchy datum for the 3D Schrödinger-Poisson system is

$$
\phi^{\mathbf{3 D}}(0, x, z)=\phi_{p, 0}(x) X_{p}^{\varepsilon}(z) .
$$

Theorem 2.7 Under Assumptions 2.2, 2.3, 2.6, let $\left(\phi_{p}^{\mathrm{SB}}, \chi_{p}^{\mathrm{SB}}, V^{\mathrm{SB}}, n^{\mathrm{SB}}\right)$ solve the quantum subband model (1.4), (1.5), (1.6) and let $\left(\psi^{3 \mathrm{D}}, V^{3 \mathrm{D}}, n^{3 \mathrm{D}}\right)$ solve the $3 D$ Schrödinger-Poisson model (1.1), (1.2). Then we have, for any $q \in(2, \infty)$ and $T>\infty$,

$$
\sup _{t \in[0, T]}\left(\left\|\phi_{p}^{\mathrm{SB}} \chi_{p}^{\mathrm{SB}}-\psi^{3 \mathrm{D}}\right\|_{L_{x, z}^{2}}+\left\|V^{\mathrm{SB}}-V^{3 \mathrm{D}}\right\|_{L_{x}^{q} L_{z}^{\infty}}+\left\|n^{\mathrm{SB}}-n^{3 \mathrm{D}}\right\|_{L_{x, z}^{1}}\right)=\mathcal{O}\left(\varepsilon^{3}\right) .
$$

Let us comment on this result and stress a specific difficulty induced by the nonlinear character of this problem. Consider first the linear equation (1.1), when the potential $V^{3 \mathrm{D}}$ is given. The adiabatic approximation of such linear Schrödinger equations is well-known and has been studied by several authors (a review of recent results can be found in [29]). Let us expand $\psi^{3 \mathrm{D}}$ on the subbands defined by (1.3), by setting $\psi^{3 \mathrm{D}}(t, x, z)=\sum_{p} \phi_{p}(t, x) \chi^{3 \mathrm{D}}(z)$. By projecting (1.1), one gets an equation for each coefficient $\phi_{p}$ :

$$
i \partial_{t} \phi_{p}=-\frac{1}{2} \Delta_{x} \phi_{p}+\epsilon_{p}^{3 \mathrm{D}} \phi_{p}+r_{p}^{\varepsilon}
$$

where the remainder is

$$
r_{p}^{\varepsilon}=-\sum_{q}\left(\frac{1}{2}\left\langle\chi_{p}^{3 \mathrm{D}} \Delta_{x} \chi_{q}^{3 \mathrm{D}}\right\rangle+i\left\langle\chi_{p}^{3 \mathrm{D}} \partial_{t} \chi_{q}^{3 \mathrm{D}}\right\rangle\right) \phi_{q}-\sum_{q}\left\langle\chi_{p}^{3 \mathrm{D}} \nabla_{x} \chi_{q}^{3 \mathrm{D}}\right\rangle \cdot \nabla_{x} \phi_{q}
$$

When $V^{3 \mathrm{D}}$ is smooth enough, this potential appears in (1.3) as a small perturbation of $V_{c}^{\varepsilon}$ and the functions $\chi_{p}^{3 \mathrm{D}}$ depend slowly on $t$ and $x$ (see Lemma A.4). Consequently, $r_{p}^{\varepsilon}$ can be proved to be small with $\varepsilon$, which shows that the dynamics is nearly diagonal. 
Unfortunately, when $V^{3 \mathrm{D}}$ is calculated selfconsistently via the Poisson equation, it is readily seen that the rapid time oscillations of the density imply that $\partial_{t} V^{3 \mathrm{D}}$ is of order $\varepsilon^{-2}$. Therefore it is not clear whether the adiabatic decoupling occurs in the nonlinear case. To circumvent this difficulty, our strategy in Section 4 consists in projecting (1.1) on the subband $\chi_{p}^{\mathrm{SB}}$ instead on $\chi_{p}^{3 \mathrm{D}}$. Indeed, in the quantum subband problem, the wavefunctions on the different modes are not quantum coherent and do not interfere. As a consequence, the density $n^{\mathrm{SB}}$ does not oscillate rapidly in time and the potential $V^{\mathrm{SB}}$ is smooth with respect to all the variables.

Remark 2.8 (The limit model) In [6], we have analyzed the behavior of the initial 3D model as $\varepsilon$ goes to 0 (for this class of initial data satisfying Assumption 2.6). We have proved that the solution of the 3D Schrödinger-Poisson model (1.1), (1.2) converges as $\varepsilon \rightarrow 0$ to the solution of the following limit model, referred to as the 2D surface density model:

$$
\begin{aligned}
i \partial_{t} \phi^{2 \mathrm{D}} & =-\frac{1}{2} \Delta_{x} \phi+W^{2 \mathrm{D}} \phi^{2 \mathrm{D}}, \\
W^{2 \mathrm{D}} & =\frac{1}{4 \pi|x|} *_{x}\left(\left|\phi^{2 \mathrm{D}}\right|^{2}\right) .
\end{aligned}
$$

In this model, the density is supported on the plane $\{z=0\}$ and $W^{2 \mathrm{D}}$ is the trace of the potential calculated by the Poisson equation with a surface density. We have proved that the error between the 3D model and the 2D limit model is of order $\mathcal{O}(\varepsilon)$, and that this order 1 in $\varepsilon$ is optimal. The quantum subband model thus appears as a better approximation of the 3D model than this limit 2D model. This corroborates the practical observations which can be done thanks to numerical experiments (such comparison was done in [27]).

\section{Well-posedness of the quantum subband model}

As we explained above, our strategy for analyzing the quantum subband model consists in solving a vectorial nonlinear Schrödinger equation

$$
i \partial_{t} \phi=-\frac{1}{2} \Delta_{x} \phi+\mathcal{F}(\phi)
$$

satisfied by $\phi=\left(\phi_{p}\right)_{p \in \mathbb{N}^{*}}$, where $\mathcal{F}(\phi)$ is defined through the resolution of a quasistatic Schrödinger-Poisson system. This proof is developed in Subsection 3.3 and relies on two crucial intermediate results:

- the well-posedness of such quasistatic Schrödinger-Poisson systems (1.5), (1.6), solved in Subsection 3.1 for given occupation factors $\left(\left|\phi^{\mathbf{S B}}\right|^{2}\right)_{p \in \mathbb{N}^{*}}$;

- an a priori estimate for the whole subband system, obtained in Subsection 3.2 thanks to the equations of mass and energy conservations. 


\subsection{The quasistatic Schrödinger-Poisson system}

In this section, as a first step towards the resolution of the whole system, we study the quasistatic part of the transient subband model, i.e. we solve the system (1.5), (1.6) for given occupation factors $\left|\phi_{p}^{\mathbf{S B}}\right|^{2}$. We rewrite this system as follows:

$$
\begin{gathered}
-\frac{1}{2} \partial_{z}^{2} \chi_{p}^{\varepsilon}+\left(V_{c}^{\varepsilon}+V^{\varepsilon}\right) \chi_{p}^{\varepsilon}=\epsilon_{p}^{\varepsilon} \chi_{p}^{\varepsilon} \quad\left(p \in \mathbb{N}^{*}\right), \\
V^{\varepsilon}=\frac{1}{4 \pi r} * \sum_{p=1}^{+\infty} \rho_{p}\left|\chi_{p}^{\varepsilon}\right|^{2}
\end{gathered}
$$

in which the surface densities $\rho_{p}(x)$, for $p \geq 1$, are given in a functional space that is precised below (possible dependences on $t$ or $\varepsilon$ are omitted in this section). It is specified that $\left(\epsilon_{p}^{\varepsilon}\right)_{p \geq 1}$ is the increasing sequence of all the eigenvalues of (3.1) and that the eigenfunctions $\chi_{p}^{\varepsilon}$ are normalized in $L^{2}(\mathbb{R})$ :

$$
\forall x \in \mathbb{R}^{2} \quad\left\langle\left|\chi_{p}^{\varepsilon}(x, z)\right|^{2}\right\rangle=1 .
$$

Before stating an existence result for this system, let us introduce a notation used throughout this paper. For any function $U \in L^{\infty}(\mathbb{R})$, and due to Assumption 2.2, the Hamiltonian $H[U]=-\frac{1}{2} \frac{d^{2}}{d z^{2}}+V_{c}^{\varepsilon}+U$ on $L^{2}(\mathbb{R})$ with the domain

$$
D(H)=\left\{\psi \in H^{2}(\mathbb{R}): \quad V_{c}^{\varepsilon} \psi \in L^{2}(\mathbb{R})\right\}
$$

admits a discrete spectrum and a complete set of eigenfunctions (see for instance $[26])$. Its eigenvalues and eigenfunctions are denoted by $\epsilon_{p}[U]$ and $\chi_{p}[U]$. Useful properties of these spectral elements are listed in the Appendix of this paper. One can note that, with these notations, the quasistatic Schrödinger-Poisson (3.1), (3.2) can be shortly reformulated under the form of a nonlinear (and nonlocal) Poisson equation:

$$
V^{\varepsilon}=\frac{1}{4 \pi r} * \sum_{p=1}^{+\infty} \rho_{p}\left|\chi_{p}\left[V^{\varepsilon}\right]\right|^{2} .
$$

In this section, we generalize some results obtained in [7], where only the first subband was considered. The following lemma was proved in this article (it is adapted here with slightly weaker assumptions):

Lemma $3.1([7])$ Let $\varepsilon>0$ be fixed and let $\rho_{1} \in L^{4 / 3}\left(\mathbb{R}^{2}\right) \cap L^{1}\left(\mathbb{R}^{2}\right)$ be such that $\rho_{1} \geq$ 0 . Assume that $\rho_{p} \equiv 0$ for $p \geq 2$. Then (3.1), (3.2) admits a unique solution such that $V^{\varepsilon} \in L^{6}\left(\mathbb{R}^{3}\right), \nabla_{x, z} V^{\varepsilon} \in L^{2}\left(\mathbb{R}^{3}\right)$. Moreover, if $\rho_{1}$ and $\widetilde{\rho_{1}}$ belong to $L^{q}\left(\mathbb{R}^{2}\right) \cap L^{1}\left(\mathbb{R}^{2}\right)$, with $q>3 / 2$, then the corresponding solutions are such that

$$
\left\|\epsilon_{1}^{\varepsilon}-\widetilde{\epsilon}_{1}^{\varepsilon}\right\|_{W^{1, \widehat{q}}\left(\mathbb{R}^{2}\right)} \leq C_{\varepsilon}\left\|\rho_{1}-\widetilde{\rho}_{1}\right\|_{q}+C_{\varepsilon}\left\|\rho_{1}-\widetilde{\rho}_{1}\right\|_{1},
$$

where $\widehat{q}=\frac{3 q}{3-q}$ if $q<3$ and $\widehat{q}=\infty$ if $q>3$, and the constant $C_{\varepsilon}$ only depends on $V_{c}$ and $\varepsilon$. 
When several subbands are occupied, the situation may be more complicated. In general, it is not clear whether the solution of this Schrödinger-Poisson system is unique (this issue was already discussed in [4], where a similar system in a bounded domain was solved). Nevertheless, this question of uniqueness finds a positive answer for a strong enough confinement potential, i.e. for $\varepsilon$ small enough. The main result of this section is the

Proposition 3.2 Let $M>0$ and $q \in(2, \infty)$. Then there exists a constant $\widetilde{\varepsilon}(M, q)>$ 0 such that, for any $\rho=\left(\rho_{p}\right)_{p \geq 1}$ belonging to $\ell^{1}\left(L^{q}\left(\mathbb{R}^{2}\right)\right) \cap \ell^{1}\left(L^{1}\left(\mathbb{R}^{2}\right)\right)$, if we have

$$
0<\varepsilon<\widetilde{\varepsilon}(M, q) \quad \text { and } \quad\|\rho\|_{\ell^{1}\left(L^{q}\left(\mathbb{R}^{2}\right)\right)}+\|\rho\|_{\ell^{1}\left(L^{1}\left(\mathbb{R}^{2}\right)\right)}<M
$$

then the Schrödinger-Poisson system (3.1), (3.2) admits a unique solution. Moreover, let $\rho=\left(\rho_{p}\right)_{p \geq 1}$ and $\widetilde{\rho}=\left(\widetilde{\rho}_{p}\right)_{p \geq 1}$ belong to $\ell^{1}\left(W^{1, q^{\#}}\left(\mathbb{R}^{2}\right)\right) \cap \ell^{1}\left(L^{1}\left(\mathbb{R}^{2}\right)\right.$ ) (with $q^{\#}$ defined by (2.1)). If $\varepsilon, \rho$ and $\widetilde{\rho}$ satisfy (3.3) then the corresponding solutions are such that

$$
\left\|\epsilon_{p}^{\varepsilon}-\widetilde{\epsilon}_{p}^{\varepsilon}\right\|_{W^{1, q}\left(\mathbb{R}^{2}\right)} \leq C_{\rho, \widetilde{\rho}}\left(\|\rho-\widetilde{\rho}\|_{\ell^{1}\left(W^{1, q^{\#}}\left(\mathbb{R}^{2}\right)\right)}+\|\rho-\widetilde{\rho}\|_{\ell^{1}\left(L^{1}\left(\mathbb{R}^{2}\right)\right)}\right),
$$

where the constant $C_{\rho, \widetilde{\rho}}$ depends on the $\ell^{1}\left(L^{1}\right)$ and $\ell^{1}\left(L^{q}\right)$ norms of $\rho$ and $\widetilde{\rho}$ but is independent of $\varepsilon$ and $p$.

Proof. Throughout this proof, $M>0$ and $q \in(2, \infty)$ are fixed. For simplicity, we shall use the short notation $\|\cdot\|$ for $\|\cdot\|_{\ell^{1}\left(L^{q}\left(\mathbb{R}^{2}\right)\right)}+\|\cdot\|_{\ell^{1}\left(L^{1}\left(\mathbb{R}^{2}\right)\right)}$.

Step 1: Existence and uniqueness. Let $\rho=\left(\rho_{p}\right)_{p \geq 1}$ be such that

$$
\|\rho\| \leq M .
$$

For $\varepsilon$ small enough, we will prove the existence and uniqueness of a solution for (3.1), (3.2) by applying the Banach fixed point theorem to a well chosen ball of $L^{\infty}\left(\mathbb{R}^{3}\right)$. To this aim, we first define a mapping $\mathcal{G}$ as follows:

$$
V \in L^{\infty}\left(\mathbb{R}^{3}\right) \longmapsto \mathcal{G}(V)=\frac{1}{4 \pi r} * \sum_{p \geq 1} \rho_{p}\left|\chi_{p}[V]\right|^{2} .
$$

Let us first check that $\mathcal{G}(V)$ belongs to $L^{\infty}\left(\mathbb{R}^{3}\right)$. Because of the normalization of the eigenfunctions, we always have $\left\|\chi_{p}[V]\right\|_{\infty, 2}=1$. Hence it is clear that $n=$ $\sum_{p \geq 1} \rho_{p}\left|\chi_{p}[V]\right|^{2}$ satisfies

$$
\|n\|_{q, 1}+\|n\|_{1} \leq\|\rho\|_{\ell^{1}\left(L^{q}\left(\mathbb{R}^{2}\right)\right)}+\|\rho\|_{\ell^{1}\left(L^{1}\left(\mathbb{R}^{2}\right)\right)} \leq M
$$

and it stems from $(2.4)$ that $\mathcal{G}(V) \in L^{\infty}\left(\mathbb{R}^{3}\right)$ and

$$
\forall V \in L^{\infty} \quad\|\mathcal{G}(V)\|_{\infty} \leq C_{0} M,
$$

where the constant $C_{0}$ is independent of $\varepsilon$ and $V$. Let

$$
\varepsilon_{0}=\left(\frac{\delta_{0}}{4 C_{0} M}\right)^{1 / 2},
$$


where $\delta_{0}$ is defined by (2.6), and

$$
\mathcal{B}=\left\{V \in L^{\infty}\left(\mathbb{R}^{3}\right): \quad\|V\|_{\infty} \leq C_{0} M\right\},
$$

where $C_{0}$ is the same constant as in (3.6). From (3.6) we deduce three facts. First, any solution of the Schrödinger-Poisson system (3.1), (3.2) (which is a fixed point of $\mathcal{G}$ ) belongs to $\mathcal{B}$. Second, $\mathcal{B}$ is stable under the action of $\mathcal{G}$. Third, for $0<\varepsilon<\varepsilon_{0}$ any $V$ in $\mathcal{B}$ satisfies (A.2).

Let $V_{1}$ and $V_{2}$ belong to $\mathcal{B}$ and $\varepsilon<\varepsilon_{0}$. Since these functions satisfy (A.2) (pointwise in $x$ ), the results of Lemma A.2 can be applied to them and it yields from (A.3):

$$
\left\|\chi_{p}\left[V_{1}\right]-\chi_{p}\left[V_{2}\right]\right\|_{\infty, 2} \leq C \varepsilon^{2}\left\|V_{1}-V_{2}\right\|_{\infty} .
$$

Then by applying again (2.4) and

$$
\mathcal{G}\left(V_{1}\right)-\mathcal{G}\left(V_{2}\right)=\frac{1}{4 \pi r} * \sum_{p \geq 1} \rho_{p}\left(\chi_{p}\left[V_{1}\right]+\chi_{p}\left[V_{2}\right]\right)\left(\chi_{p}\left[V_{1}\right]-\chi_{p}\left[V_{2}\right]\right)
$$

we obtain

$$
\left\|\mathcal{G}\left(V_{1}\right)-\mathcal{G}\left(V_{2}\right)\right\|_{\infty} \leq C_{1} \varepsilon^{2} M\left\|V_{1}-V_{2}\right\|_{\infty},
$$

where $C_{1}$ only depends on $V_{c}$. By choosing

$$
\widetilde{\varepsilon}(M, q):=\min \left(\varepsilon_{0}, \frac{1}{2}\left(C_{1} M\right)^{-1 / 2}\right),
$$

we ensure that for $\varepsilon<\widetilde{\varepsilon}(M, q)$ the mapping $\mathcal{G}$ is a contraction on $\mathcal{B}$, thus admits a unique fixed point. This proves the first part of Proposition 3.2. From now on, we assume that $\varepsilon<\widetilde{\varepsilon}(M, q)$.

Step 2: Lipschitz continuity in $L_{x}^{s} L_{z}^{\infty}$. Consider two sequences of occupation factors $\rho=\left(\rho_{p}\right)_{p \geq 1}$ and $\widetilde{\rho}=\left(\widetilde{\rho_{p}}\right)_{p \geq 1}$ in $\ell^{1}\left(L^{1}\left(\mathbb{R}^{2}\right)\right) \cap \ell^{1}\left(L^{q}\left(\mathbb{R}^{2}\right)\right)$ satisfying (3.5) and let $V^{\varepsilon}, \widetilde{V}^{\varepsilon}$ be the corresponding solutions of (3.1), (3.2). We have

$$
V^{\varepsilon}-\widetilde{V^{\varepsilon}}=\delta V_{1}+\delta V_{2}
$$

with

$$
\delta V_{1}=\frac{1}{4 \pi r} * \sum_{p \geq 1}\left(\rho_{p}-\widetilde{\rho}_{p}\right)\left|\chi_{p}\left[\widetilde{V}^{\varepsilon}\right]\right|^{2} \quad ; \quad \delta V_{2}=\frac{1}{4 \pi r} * \sum_{p \geq 1} \rho_{p}\left(\left|\chi_{p}\left[V^{\varepsilon}\right]\right|^{2}-\left|\chi_{p}\left[\tilde{V}^{\varepsilon}\right]\right|^{2}\right) .
$$

Since we have $q>2$, by (2.4) and the normalization of the eigenfunctions $\chi_{p}$ in $L_{x}^{\infty} L_{z}^{2}$, we get

$$
\left\|\delta V_{1}\right\|_{\infty} \leq C\|\rho-\widetilde{\rho}\|_{\ell^{1}\left(L^{q}\left(\mathbb{R}^{2}\right)\right)}^{\theta}\|\rho-\widetilde{\rho}\|_{\ell^{1}\left(L^{1}\left(\mathbb{R}^{2}\right)\right)}^{1-\theta} \leq C\|\rho-\widetilde{\rho}\| .
$$

Furthermore, (A.3) implies that, for $\alpha \in[1, q]$,

$$
\begin{aligned}
\left\|\sum_{p \geq 1} \rho_{p}\left(\left|\chi_{p}\left[V^{\varepsilon}\right]\right|^{2}-\left|\chi_{p}\left[\widetilde{V}^{\varepsilon}\right]\right|^{2}\right)\right\|_{\alpha, 1} & \leq \sum_{p \geq 1}\left\|\rho_{p}\right\|_{\alpha}\left\|\chi_{p}\left[V^{\varepsilon}\right]-\chi_{p}\left[\widetilde{V}^{\varepsilon}\right]\right\|_{\infty, 2} \\
& \leq C \varepsilon^{2}\|\rho\|\left\|V^{\varepsilon}-\widetilde{V}^{\varepsilon}\right\|_{\infty} .
\end{aligned}
$$


By applying again (2.4), we deduce

$$
\left\|\delta V_{2}\right\|_{\infty} \leq C \varepsilon^{2} M\left\|V^{\varepsilon}-\widetilde{V}^{\varepsilon}\right\|_{\infty}
$$

and finally

$$
\left\|V^{\varepsilon}-\widetilde{V}^{\varepsilon}\right\|_{\infty} \leq C\|\rho-\widetilde{\rho}\|+C \varepsilon^{2} M\left\|V^{\varepsilon}-\widetilde{V}^{\varepsilon}\right\|_{\infty} .
$$

Thus, up to a modification of $\widetilde{\varepsilon}(M, q)$, we obtain the Lipschitz estimate in $L^{\infty}\left(\mathbb{R}^{3}\right)$ :

$$
\left\|V^{\varepsilon}-\widetilde{V}^{\varepsilon}\right\|_{\infty} \leq C\|\rho-\widetilde{\rho}\| .
$$

Let us now choose an $s \in(2, \infty)$. By using interpolation inequalities, (3.7) and (2.2), it is readily seen that we have a similar estimate of $V^{\varepsilon}-\widetilde{V}^{\varepsilon}$ in $L_{x}^{s} L_{z}^{\infty}$. One can sum up this Step 2 in the following result:

$$
\forall s \in(2, \infty] \quad\left\|V^{\varepsilon}-\widetilde{V}^{\varepsilon}\right\|_{s, \infty}+\frac{1}{\varepsilon^{2}}\left\|\chi_{p}\left[V^{\varepsilon}\right]-\chi_{p}\left[\widetilde{V}^{\varepsilon}\right]\right\|_{s, 2} \leq C\|\rho-\widetilde{\rho}\|,
$$

where $C$ depends on $s$ but not on $\varepsilon, p$.

Step 3: estimating the gradients. Consider now a sequence of occupation factors $\rho=\left(\rho_{p}\right)_{p \geq 1}$ in $\ell^{1}\left(L^{1}\left(\mathbb{R}^{2}\right)\right) \cap \ell^{1}\left(W^{1, q^{\#}}\left(\mathbb{R}^{2}\right)\right)$, where $q^{\#}=\frac{2 q}{2+q}$. A Sobolev embedding implies that $\rho \in \ell^{1}\left(L^{q}\left(\mathbb{R}^{2}\right)\right)$. We assume that (3.5) is satisfied and consider the unique solution $V^{\varepsilon}$ of (3.1), (3.2). As in the proof of Lemma A.2 (see (A.5)), since $V^{\varepsilon}$ satisfies (A.2) we can show that

$$
\forall p \in \mathbb{N}^{*}, \quad \forall k \in \mathbb{N}^{*} \backslash\{p\}, \quad\left|\epsilon_{p}\left[V^{\varepsilon}\right]-\epsilon_{k}\left[V^{\varepsilon}\right]\right| \geq \frac{\delta_{0}}{2 \varepsilon^{2}},
$$

where $\delta_{0}>0$. From Lemma A.1, we deduce that

$$
\left\|\nabla_{x} \chi_{p}\left[V^{\varepsilon}\right]\right\|_{q, 2}=\left\|\sum_{k \neq p} \frac{\left\langle\chi_{p}\left[V^{\varepsilon}\right] \chi_{k}\left[V^{\varepsilon}\right] \nabla_{x} V^{\varepsilon}\right\rangle}{\epsilon_{p}\left[V^{\varepsilon}\right]-\epsilon_{k}\left[V^{\varepsilon}\right]} \chi_{k}\left[V^{\varepsilon}\right]\right\|_{q, 2} \leq C \varepsilon^{2}\left\|\nabla_{x} V^{\varepsilon}\right\|_{q, \infty},
$$

where we used the fact that $\left(\chi_{k}\right)_{k \geq 1}$ is an orthonormal basis. Hence $n^{\varepsilon}=$ $\sum_{p \geq 1} \rho_{p}\left|\chi_{p}\left[V^{\varepsilon}\right]\right|^{2}$ is such that

$$
\left\|\nabla_{x} n^{\varepsilon}\right\|_{q^{\#, 1}} \leq\|\rho\|_{\ell^{1}\left(W^{1, q} q^{\#}\left(\mathbb{R}^{2}\right)\right)}+C M \varepsilon^{2}\left\|\nabla_{x} V^{\varepsilon}\right\|_{q, \infty} .
$$

Here we have used a Hölder inequality to estimate:

$$
\left\|\rho_{p} \chi_{p}^{\varepsilon} \nabla_{x} \chi_{p}^{\varepsilon}\right\|_{q^{\#}, 1} \leq\left\|\rho_{p}\right\|_{2}\left\|\chi_{p}^{\varepsilon}\right\|_{\infty, 2}\left\|\nabla_{x} \chi_{p}^{\varepsilon}\right\|_{q, 2} .
$$

By (2.3) and the Poisson equation (3.2), we get

$$
\left\|\nabla_{x, z} V^{\varepsilon}\right\|_{q, \infty} \leq C\|\rho\|_{\ell^{1}\left(W^{1, q^{\#}}\left(\mathbb{R}^{2}\right)\right)}+C M \varepsilon^{2}\left\|\nabla_{x} V^{\varepsilon}\right\|_{q, \infty}
$$

then, (up to another modification of $\widetilde{\varepsilon}(M, q)$ ), we deduce that, for $\varepsilon<\widetilde{\varepsilon}(M, q)$,

$$
\left\|\nabla_{x, z} V^{\varepsilon}\right\|_{q, \infty}+\frac{1}{\varepsilon^{2}}\left\|\nabla_{x} \chi_{p}^{\varepsilon}\right\|_{q, 2} \leq C\|\rho\|_{\ell^{1}\left(W^{1, q^{\#}}\left(\mathbb{R}^{2}\right)\right)} .
$$


Step 4: Lipschitz continuity of the gradients. From (A.4) and the chain rule, we first deduce the following useful inequality. For any $V_{1}, V_{2}$ in $L^{\infty}\left(\mathbb{R}^{3}\right)$ such that $\nabla_{x} V_{1}$ and $\nabla_{x} V_{2}$ belong to $L_{x}^{q} L_{z}^{\infty}$, we have

$$
\left\|\nabla_{x} \chi_{p}\left[V_{1}\right]-\nabla_{x} \chi_{p}\left[V_{2}\right]\right\|_{q, 2} \leq C_{V_{1}, V_{2}} \varepsilon^{4}\left\|V_{1}-V_{2}\right\|_{\infty}+C \varepsilon^{2}\left\|\nabla_{x} V_{1}-\nabla_{x} V_{2}\right\|_{q, \infty}
$$

where $C_{V_{1}, V_{2}}$ depends on the $L_{x}^{q} L_{z}^{\infty}$ norms of $\nabla_{x} V_{1}$ and $\nabla_{x} V_{2}$ and $C$ is independent of $V_{1}$ and $V_{2}$.

Consider now two sequences of occupation factors $\rho=\left(\rho_{p}\right)_{p \geq 1}$ and $\widetilde{\rho}=\left(\widetilde{\rho_{p}}\right)_{p \geq 1}$ in $\ell^{1}\left(L^{1}\left(\mathbb{R}^{2}\right)\right) \cap \ell^{1}\left(W^{1, q^{\#}}\left(\mathbb{R}^{2}\right)\right)$, both satisfying (3.5) and let $V^{\varepsilon}, \widetilde{V}^{\varepsilon}$ be the corresponding solutions. By applying (3.10), (2.3), by using the bounds (3.8), (3.9), and by estimating each term in the right-hand side of

$$
\begin{aligned}
\nabla_{x} n^{\varepsilon}-\nabla_{x} \widetilde{n}^{\varepsilon}= & \sum_{p}\left(\nabla_{x} \rho_{p}-\nabla_{x} \widetilde{\rho_{p}}\right)\left|\chi_{p}^{\varepsilon}\right|^{2}+2 \sum_{p}\left(\rho_{p}-{\widetilde{\rho_{p}}}\right) \chi_{p}^{\varepsilon} \nabla_{x} \chi_{p}^{\varepsilon} \\
& +\sum_{p} \nabla_{x} \widetilde{\rho_{p}}\left(\left|\chi_{p}^{\varepsilon}\right|^{2}-\left|\widetilde{\chi}_{p}^{\varepsilon}\right|^{2}\right)+\sum_{p} \widetilde{\rho_{p}} \nabla_{x}\left(\left|\chi_{p}^{\varepsilon}\right|^{2}-\left|\widetilde{\chi}_{p}^{\varepsilon}\right|^{2}\right),
\end{aligned}
$$

we obtain (up to another modification of $\widetilde{\varepsilon}(M, q)$ )

$$
\left\|\nabla_{x, z} V^{\varepsilon}-\nabla_{x, z} \widetilde{V}^{\varepsilon}\right\|_{q, \infty} \leq C_{\rho, \widetilde{\rho}}\left(\|\rho-\widetilde{\rho}\|_{\ell^{1}\left(W^{1, q^{\#}}\left(\mathbb{R}^{2}\right)\right)}+\|\rho-\widetilde{\rho}\|_{\ell^{1}\left(L^{1}\left(\mathbb{R}^{2}\right)\right)}\right) .
$$

Finally, (3.4) can be deduced from this estimate, by using (A.1), (3.8) and

$\nabla_{x} \epsilon_{p}\left[V^{\varepsilon}\right]-\nabla_{x} \epsilon_{p}\left[\widetilde{V}^{\varepsilon}\right]=\left\langle\left(\nabla_{x} V^{\varepsilon}-\nabla_{x} \widetilde{V}^{\varepsilon}\right)\left|\chi_{p}\left[V^{\varepsilon}\right]\right|^{2}\right\rangle+\left\langle\nabla_{x} \widetilde{V}^{\varepsilon}\left(\left|\chi_{p}\left[V^{\varepsilon}\right]\right|^{2}-\left|\chi_{p}\left[\widetilde{V}^{\varepsilon}\right]\right|^{2}\right)\right\rangle$.

Remark 3.3 In the special case of decreasing occupation factors, i.e. if, pointwise in $x$ and for all $p \geq 1$, we have $\rho_{p}(x) \geq \rho_{p+1}(x)$, then one can show that (3.1), (3.2) admits a unique solution for any $\varepsilon>0$ (arbitrarily large). Lemma 3.1 is a particular case of this situation. Inspired by $[21,22,4,7]$, the proof of this statement can be based on a variational argument, by introducing the functional

$$
J(V)=\frac{1}{2} \iint_{\mathbb{R}^{3}}\left|\nabla_{x, z} V\right|^{2} d x d z-\sum_{p \geq 1} \int_{\mathbb{R}^{2}} \rho_{p}\left(\epsilon_{p}[V]-\epsilon_{p}[0]\right) d x
$$

on $\mathcal{K}=\left\{V \in L^{6}\left(\mathbb{R}^{3}\right): \quad \nabla_{x, z} V \in L^{2}\left(\mathbb{R}^{3}\right)\right\}$. One can check that this functional is continuous, coercive on $\mathcal{K}$ and that its first and second derivatives are given by

$$
\begin{aligned}
& \partial_{V} J \cdot W=\iint_{\mathbb{R}^{3}}-\left(\Delta_{x, z} V\right) W d x d z-\sum_{p \geq 1} \iint_{R R^{3}} \rho_{p}\left|\chi_{p}[V]\right|^{2} W d x d z, \\
& \partial_{V}^{2} J[V] \cdot W \cdot W= \iint_{\mathbb{R}^{3}}\left|\nabla_{x, z} W\right|^{2} d x d z \\
&-\sum_{p} \sum_{q \neq p} \int_{\mathbb{R}^{2}} \frac{\rho_{p}(x)-\rho_{q}(x)}{\epsilon_{p}[V]-\epsilon_{q}[V]}\left\langle\chi_{p}[V] \chi_{q}[V] W\right\rangle^{2} \geq 0 .
\end{aligned}
$$

which means that the unique minimizer of this strictly convex functional solves the Schrödinger-Poisson system (3.1), (3.2). 


\subsection{A priori estimates}

Since the 2D Schrödinger equations (1.4) are decoupled, the total mass is obviously conserved by the quantum subband model (1.4), (1.5), (1.6):

$$
\forall t \geq 0 \quad \sum_{p \geq 1}\left\|\phi_{p}^{\mathrm{SB}}(t)\right\|_{2}^{2}=\sum_{p \geq 1}\left\|\phi_{p, 0}\right\|_{2}^{2}
$$

Another important property of this model is the energy conservation. Let us define the total energy by

$$
\mathcal{E}_{\text {tot }}=\mathcal{E}_{\text {kin }}+\mathcal{E}_{\text {pot }}
$$

where the kinetic energy is the sum of the kinetic energies along $x$ and $z$ :

$$
\mathcal{E}_{k i n}=\frac{1}{2} \int_{\mathbb{R}^{2}} \sum_{p \geq 1}\left|\nabla_{x} \phi_{p}^{\mathrm{SB}}\right|^{2} d x+\frac{1}{2} \iint_{\mathbb{R}^{3}} \sum_{p \geq 1}\left|\phi_{p}^{\mathrm{SB}}\right|^{2}\left|\partial_{z} \chi_{p}^{\mathrm{SB}}\right|^{2} d x d z
$$

and the potential energy is the sum of the selfconsistent potential energy and the external potential energy:

$$
\mathcal{E}_{p o t}=\frac{1}{2} \iint_{\mathbb{R}^{3}}\left|\nabla_{x, z} V^{\mathrm{SB}}\right|^{2} d x d z+\iint_{\mathbb{R}^{3}} \sum_{p \geq 1} V_{c}^{\varepsilon}\left|\phi_{p}^{\mathrm{SB}}\right|^{2}\left|\chi_{p}^{\mathrm{SB}}\right|^{2} d x d z
$$

This total energy is a conserved quantity, as stated in the

Proposition 3.4 Let $\varepsilon>0$ be fixed. Let $\left(\phi_{p}^{\mathrm{SB}}\right)_{p \geq 1}$ be a solution of (1.4), (1.5), (1.6) such that for all $p \in \mathbb{N}^{*}$ we have $\phi_{p}^{\mathrm{SB}} \in C\left(\mathbb{R}_{+}, H^{1}\left(\mathbb{R}^{2}\right)\right)$ and such that $\mathcal{E}_{\text {tot }}(0)<+\infty$. Then the total energy is independent of time:

$$
\forall t \in \mathbb{R}_{+} \quad \mathcal{E}_{t o t}(t)=\mathcal{E}_{\text {tot }}(0) .
$$

Proof. The technique to obtain this energy estimate is the same as for the standard Schrödinger-Poisson system $[11,19]$. We shall only prove it formally and do not develop the rigourous justification of the calculations (which can be done by a classical regularization procedure). By multiplying (1.4) by $\partial_{t} \overline{\phi_{p}^{\mathbf{S B}}}$, integrating on $\mathbb{R}^{2}$, summing for $p \in \mathbb{N}^{*}$ and taking the real part of the so-obtained equation, we get

$$
\frac{d}{d t}\left(\frac{1}{2} \int_{\mathbb{R}^{2}} \sum_{p \geq 1}\left|\nabla_{x} \phi_{p}^{\mathrm{SB}}\right|^{2} d x+\int_{\mathbb{R}^{2}} \sum_{p \geq 1} \epsilon_{p}^{\mathrm{SB}}\left|\phi_{p}^{\mathrm{SB}}\right|^{2} d x\right)=\int_{\mathbb{R}^{2}} \sum_{p \geq 1} \partial_{t} \epsilon_{p}^{\mathrm{SB}}\left|\phi_{p}^{\mathrm{SB}}\right|^{2} d x
$$

Since $\epsilon_{p}^{\mathrm{SB}}, \chi_{p}^{\mathrm{SB}}$ are the eigen-elements of $-\frac{1}{2} \partial_{z}^{2}+V_{c}^{\varepsilon}+V^{\mathrm{SB}}$, we have

$$
\epsilon_{p}^{\mathrm{SB}}=\frac{1}{2} \int_{\mathbb{R}}\left|\partial_{z} \chi_{p}^{\mathrm{SB}}\right|^{2} d z+\int_{\mathbb{R}}\left(V_{c}^{\varepsilon}+V^{\mathrm{SB}}\right)\left|\chi_{p}^{\mathrm{SB}}\right|^{2} d z
$$

and Lemma A.1 gives

$$
\partial_{t} \epsilon_{p}^{\mathrm{SB}}=\int_{\mathbb{R}}\left|\chi_{p}^{\mathrm{sB}}\right|^{2} \partial_{t} V^{\mathrm{SB}} d z
$$


By substituting these equations in (3.12) and by using the Poisson equation (1.6), one obtains the result.

Thanks to this energy conservation, we will now obtain an a priori estimate independent of $\varepsilon$, which will be used in the next section to prove that the solution of the quantum subband model is global in time. Although all the terms defining $\mathcal{E}_{\text {tot }}$ are nonnegative, the fact that the energy conservation provides such an $a$ priori estimate is not obvious. Indeed, due to the confinement potential energy $\iint_{\mathbb{R}^{3}} \sum_{p \geq 1} V_{c}^{\varepsilon}\left|\phi_{p}^{\mathrm{SB}}\right|^{2}\left|\chi_{p}^{\mathrm{SB}}\right|^{2} d x d z$, the total energy at the initial time $\mathcal{E}_{\text {tot }}(0)$ is of order $\mathcal{O}\left(\frac{1}{\varepsilon^{2}}\right)$, thus the total energy remains unbounded for all time.

Proposition 3.5 Let $\left(\phi_{p, 0}\right)_{p \geq 1}$ satisfy Assumption 2.4 and let $\left(\phi_{p}^{\mathrm{SB}}\right)_{p \geq 1}$ be a solution of (1.4), (1.5), (1.6) such that for all $p \in \mathbb{N}^{*}$ and $\varepsilon>0$ we have $\phi_{p}^{\mathrm{SB}} \in C\left(\mathbb{R}_{+}, H^{1}\left(\mathbb{R}^{2}\right)\right)$. Then there exists a continuous function $\mathcal{C}(\cdot)$ independent of $\varepsilon$ such that we have, for any $t \in[0,+\infty)$,

$$
\sum_{p \geq 1}\left\|\phi_{p}^{\mathbf{S B}}\right\|_{H^{1}\left(\mathbb{R}^{2}\right)}^{2} \leq \mathcal{C}\left(\sum_{p \geq 1}\left\|\phi_{p, 0}\right\|_{H^{1}\left(\mathbb{R}^{2}\right)}^{2}\right)
$$

Proof. A first easy estimate comes from the conservation of the $L^{2}$ norm for each 2D Schrödinger equation (1.4):

$$
\forall t \geq 0 \quad \sum_{p \geq 1} \frac{E_{p}}{\varepsilon^{2}}\left\|\phi_{p}^{\mathrm{SB}}(t)\right\|_{L^{2}\left(\mathbb{R}^{2}\right)}^{2}=\sum_{p \geq 1} \frac{E_{p}}{\varepsilon^{2}}\left\|\phi_{p, 0}\right\|_{L^{2}\left(\mathbb{R}^{2}\right)}^{2}<\infty .
$$

At any time $t \geq 0$, the total energy can be reformulated thanks to (3.13) and the Poisson equation as follows:

$$
\mathcal{E}_{\text {tot }}=\frac{1}{2} \sum_{p \geq 1}\left\|\nabla_{x} \phi_{p}^{\mathrm{SB}}\right\|_{2}^{2}+\int_{\mathbb{R}^{2}} \sum_{p \geq 1} \epsilon_{p}^{\mathrm{SB}}\left|\phi_{p}^{\mathrm{SB}}\right|^{2} d x-\frac{1}{2} \iint_{\mathbb{R}^{3}} V^{\mathrm{SB}}\left|\phi_{p}^{\mathrm{SB}}\right|^{2}\left|\chi_{p}^{\mathrm{SB}}\right|^{2} d x d z .
$$

The conservation of the total energy stated in Proposition 3.4 is then rewritten:

$$
\begin{aligned}
\sum_{p \geq 1} & \left\|\nabla_{x} \phi_{p}^{\mathrm{SB}}(t)\right\|_{2}^{2}=\sum_{p \geq 1}\left\|\nabla_{x} \phi_{p, 0}\right\|_{2}^{2} \\
& +2 \int_{\mathbb{R}^{2}} \sum_{p \geq 1}\left(\frac{E_{p}}{\varepsilon^{2}}-\epsilon_{p}^{\mathrm{SB}}(t)\right)\left|\phi_{p}^{\mathrm{SB}}\right|^{2}(t) d x-2 \int_{\mathbb{R}^{2}} \sum_{p \geq 1}\left(\frac{E_{p}}{\varepsilon^{2}}-\epsilon_{p}^{\mathrm{SB}}(0)\right)\left|\phi_{p, 0}\right|^{2}(t) d x \\
& +\iint_{\mathbb{R}^{3}}\left(V^{\mathrm{SB}}\left|\phi_{p}^{\mathrm{SB}}\right|^{2}\left|\chi_{p}^{\mathrm{SB}}\right|^{2}\right)(t) d x d z-\iint_{\mathbb{R}^{3}}\left(V^{\mathrm{SB}}\left|\phi_{p}^{\mathrm{SB}}\right|^{2}\left|\chi_{p}^{\mathrm{SB}}\right|^{2}\right)(0) d x d z,
\end{aligned}
$$

where we used (3.14). By applying (A.1), we obtain now

$$
\left|\epsilon_{p}^{\mathrm{SB}}-\frac{E_{p}}{\varepsilon^{2}}\right| \leq\left\|V^{\mathrm{SB}}\right\|_{\infty}
$$

Therefore, it stems from (3.11), (3.15) and Assumption 2.4 that

$$
\sum_{p \geq 1}\left\|\nabla_{x} \phi_{p}^{\mathrm{SB}}(t)\right\|_{2}^{2} \leq C+C\left\|V^{\mathrm{sB}}(t)\right\|_{\infty}+C\left\|V^{\mathrm{SB}}(0)\right\|_{\infty},
$$


where (in this proof) $C$ denotes a generic constant depending only on $\sum_{p \geq 1}\left\|\phi_{p, 0}\right\|_{H^{1}\left(\mathbb{R}^{2}\right)}^{2}$.

It remains to estimate the $L^{\infty}$ norm of $V^{\mathrm{sB}}$. A Gagliardo-Nirenberg inequality in dimension 2 yields, for any $q \in[2, \infty)$ and $t \geq 0$,

$$
\left\|\phi_{p}^{\mathrm{SB}}(t)\right\|_{q} \leq C\left\|\phi_{p}^{\mathrm{SB}}(t)\right\|_{2}^{2 / q}\left\|\nabla_{x} \phi_{p}^{\mathrm{SB}}(t)\right\|_{2}^{(q-2) / q} .
$$

Thus, by using a Hölder inequality, we get for any $q \in[1, \infty)$

$$
\sum_{p \geq 1}\left\|\rho_{p}^{\mathrm{SB}}(t)\right\|_{q} \leq C\left(\sum_{p \geq 1}\left\|\phi_{p}^{\mathrm{SB}}(t)\right\|_{2}^{2}\right)^{1 / q}\left(\sum_{p \geq 1}\left\|\nabla_{x} \phi_{p}^{\mathrm{SB}}(t)\right\|_{2}^{2}\right)^{(q-1) / q}
$$

where we have set $\rho_{p}^{\mathrm{SB}}=\left|\phi_{p}^{\mathrm{SB}}\right|^{2}$. From (3.11) and the fact that the $\chi_{p}^{\mathrm{SB}}$ 's are normalized in $L_{z}^{2}(\mathbb{R})$, we deduce that the density defined by $n^{\mathrm{SB}}=\sum_{p \geq 1} \rho_{p}^{\mathrm{SB}}\left|\chi_{p}^{\mathrm{SB}}\right|^{2}$ satisfies, for any $q \in[1, \infty)$,

$$
\left\|n^{\mathrm{SB}}(t)\right\|_{q, 1} \leq C\left(\sum_{p \geq 1}\left\|\nabla_{x} \phi_{p}^{\mathrm{SB}}(t)\right\|_{2}^{2}\right)^{(q-1) / q}
$$

By choosing for $q$ any real number in $(2, \infty)$, we deduce from the Poisson equation (1.6) and from (2.4) that

$$
\left\|V^{\mathrm{SB}}(t)\right\|_{\infty} \leq C\left(\sum_{p \geq 1}\left\|\nabla_{x} \phi_{p}^{\mathrm{SB}}(t)\right\|_{2}^{2}\right)^{1 / 2}
$$

With Assumption 2.4, this implies $\left\|V^{\mathrm{sB}}(0)\right\|_{\infty} \leq C$. Moreover, by substituting this estimate in (3.16), we get

$$
\sum_{p \geq 1}\left\|\nabla_{x} \phi_{p}^{\mathbf{S B}}(t)\right\|_{2}^{2} \leq C+C\left(\sum_{p \geq 1}\left\|\nabla_{x} \phi_{p}^{\mathrm{SB}}(t)\right\|_{2}^{2}\right)^{1 / 2}
$$

which enables to complete the proof.

\subsection{Proof of Theorem 2.5}

With the two previous subsections, we have the tools to show that the quantum subband model is well-posed and to prove Theorem 2.5 announced in Subsection 2.2. In fact, by performing the change of unknown

$$
\widetilde{\phi_{p}^{\mathbf{S B}}}=e^{i E_{p} t / \varepsilon^{2}} \phi_{p}^{\mathbf{S B}},
$$

it is readily seen that $(1.4),(1.5),(1.6)$ is equivalent to

$$
i \partial_{t} \widetilde{\phi_{p}^{\mathrm{SB}}}=-\frac{1}{2} \Delta_{x} \widetilde{\phi_{p}^{\mathrm{SB}}}+\left(\epsilon_{p}^{\mathrm{SB}}-\frac{E_{p}}{\varepsilon^{2}}\right) \widetilde{\phi_{p}^{\mathrm{SB}}}
$$


coupled to the same equations (1.5), (1.6).

Let $M>0$ and $q \in(2, \infty)$ be fixed. Introduce the Hilbert space $\mathcal{H}=\ell^{2}\left(H^{1}\left(\mathbb{R}^{2}\right)\right)$ and let $\phi=\left(\phi_{p}\right)_{p \geq 1} \in \mathcal{H}$. Sobolev embeddings and interpolation estimates imply that the sequence of the $\rho_{p}$ 's defined by $\rho_{p}=\left|\phi_{p}\right|^{2}$ is bounded as follows:

$$
\|\rho\|_{\ell^{1}\left(W^{1, q^{\#}}\left(\mathbb{R}^{2}\right)\right)}+\|\rho\|_{\ell^{1}\left(L^{q}\left(\mathbb{R}^{2}\right)\right)}+\|\rho\|_{\ell^{1}\left(L^{1}\left(\mathbb{R}^{2}\right)\right)} \leq C_{0}\|\phi\|_{\mathcal{H}}^{2},
$$

with $q^{\#}$ defined by (2.1). We set

$$
\varepsilon(M, q)=\widetilde{\varepsilon}\left(2 C_{0} \mathcal{C}(M), q\right),
$$

where the function $\widetilde{\varepsilon}(\cdot, q)$ is defined in Proposition 3.2 and the function $\mathcal{C}(\cdot)$ is defined in Proposition 3.5. All along this proof, the parameter $\varepsilon$ is taken such that $\varepsilon<$ $\varepsilon(M, q)$. Denoting

$$
\mathcal{B}_{M}=\left\{\phi \in \mathcal{H}: \quad\|\phi\|_{\mathcal{H}}<\sqrt{2 \mathcal{C}(M)}\right\},
$$

(3.18) ensures that $\varepsilon$ and the sequence $\rho$ corresponding to any $\phi \in \mathcal{B}_{M}$ satisfy

$$
0<\varepsilon<\widetilde{\varepsilon}\left(2 C_{0} \mathcal{C}(M), q\right) \quad \text { and } \quad\|\rho\|_{\ell^{1}\left(L^{q}\left(\mathbb{R}^{2}\right)\right)}+\|\rho\|_{\ell^{1}\left(L^{1}\left(\mathbb{R}^{2}\right)\right)}<2 C_{0} \mathcal{C}(M) .
$$

Consequently, by Proposition 3.2, the Schrödinger-Poisson system (3.1), (3.2) associated to this sequence of occupation factors is well-posed.

In order to prove the local-in-time existence of a unique weak solution to (1.4), (1.5), (1.6), let us reformulate this system as a nonlinear evolution problem on $\mathcal{H}$. We introduce the following function on $\mathcal{B}_{M}$ :

$$
\phi=\left(\phi_{p}\right)_{p \geq 1} \longmapsto \mathcal{F}(\phi)=\left(\left(\epsilon_{p}^{\varepsilon}-\frac{E_{p}}{\varepsilon^{2}}\right) \phi_{p}\right)_{p \geq 1}
$$

where $\epsilon_{p}^{\varepsilon}$ is (uniquely) defined through the resolution of the Schrödinger-Poisson problem (3.1), (3.2) associated to the sequence $\left(\left|\phi_{p}\right|^{2}\right)_{p \geq 1}$. From (3.4), (3.18) and a Sobolev embedding, we deduce that for any $\phi \in \mathcal{B}_{M}$ there exists a constant $C$ independent of $p$ and $\varepsilon$ such that

$$
\forall p \in \mathbb{N}^{*} \quad\left\|\epsilon_{p}^{\varepsilon}-\frac{E_{p}}{\varepsilon^{2}}\right\|_{\infty} \leq C, \quad\left\|\nabla_{x} \epsilon_{p}^{\varepsilon}\right\|_{q} \leq C .
$$

Then it is clear with the Sobolev embedding $H^{1}\left(\mathbb{R}^{2}\right) \subset L^{q^{*}}\left(\mathbb{R}^{2}\right)$ that, for any $\phi \in \mathcal{B}_{M}$, we have $\mathcal{F}(\phi) \in \mathcal{H}$. Similarly, (3.4) implies that $\mathcal{F}$ is Lipschitz continuous on $\mathcal{B}_{M}$. This is enough to apply a standard theorem [24] for the following nonlinear evolutions equation on $\mathcal{H}$ :

$$
i \partial_{t} \phi=-\frac{1}{2} \Delta_{x} \phi+\mathcal{F}(\phi),
$$

which is equivalent to (3.17), (1.5), (1.6). If the initial datum satisfies

$$
\sum_{p \geq 1}\left\|\phi_{p, 0}\right\|_{H^{1}}^{2}<M
$$


then there exists a maximal time $T \in(0,+\infty]$ and a solution $\phi \in C([0, T), \mathcal{H})$ of this problem, which is such that

$$
\text { if } \quad T<+\infty \quad \text { then } \quad \lim _{t \rightarrow T}\|\phi(t)\|_{\mathcal{H}}^{2}=2 \mathcal{C}(M) .
$$

Besides, Proposition 3.5 shows that

$$
\forall t \in[0, T) \quad\|\phi(t)\|_{\mathcal{H}}^{2}<\mathcal{C}(M) .
$$

Therefore, we have necessarily $T=+\infty$ and the solution is global in time. The estimates in Theorem 2.5 can be obtained directly by using (3.4), (3.8) with $\widetilde{\rho}=0$ and (3.9).

Special case: initial data on the ground state. In the special case where only the first subband is occupied initially, let us check that $\varepsilon$ can be arbitrary. Since the 2D Schrödinger equations (1.4) are decoupled, for any $t \geq 0$ and $p \geq 2$ we will have $\phi_{p}^{\varepsilon}(t) \equiv 0$. Hence one can reproduce the above proof by using Lemma 3.1 instead of Proposition 3.2 for the resolution of the quasistatic Schrödinger-Poisson (with no restriction on $\varepsilon$ ). We define the following function on $H^{1}\left(\mathbb{R}^{2}\right)$ :

$$
\phi_{1} \longmapsto \mathcal{F}_{1}\left(\phi_{1}\right)=\left(\epsilon_{1}^{\varepsilon}-\frac{E_{1}}{\varepsilon^{2}}\right) \phi_{1} .
$$

Thanks to Lemma 3.1 (by choosing $q=12 / 7$ ) and Sobolev embeddings, for any $\varepsilon$ and for any functions $\phi_{1}$ and $\widetilde{\phi}_{1}$, the corresponding solutions of (3.1), (3.2) satisfy

$$
\left\|\epsilon_{1}-\widetilde{\epsilon}_{1}\right\|_{\infty}+\left\|\nabla_{x} \epsilon_{1}-\nabla_{x} \widetilde{\epsilon}_{1}\right\|_{4} \leq C_{\varepsilon}\left\|\phi_{1}-\widetilde{\phi}_{1}\right\|_{H^{1}\left(\mathbb{R}^{2}\right)},
$$

where $C_{\varepsilon}$ depends on $\varepsilon$ and the functions itself $\phi_{1}$ and $\widetilde{\phi_{1}}$. Therefore, the functional $\mathcal{F}_{1}$ is locally Lipschitz continuous on $H^{1}\left(\mathbb{R}^{2}\right)$, which enables to complete the proof of global existence and uniqueness.

\section{Asymptotic analysis}

The aim of this section is to estimate the discrepancy between the solutions of quantum subband model studied above in this paper and the 3D Schrödinger-Poisson model. From now on, in accordance with Assumption 2.6, it is assumed that only the $p$ th subband is populated ( $p$ is a given fixed integer): $\phi_{k} \equiv 0$ for $k \neq p$. Moreover, in this part, $M>0$ and $q \in(2, \infty)$ are fixed (with $q^{\prime}, q^{*}$ and $q^{\#}$ still defined by (2.1)). We always assume that the conditions of existence and uniqueness for (1.4), (1.5), (1.6) specified in Theorem 2.5 are satisfied (i.e. that $\varepsilon$ is small enough).

In order to estimate the difference between these models, we will need some estimates independent of $\varepsilon$. In the case of the quantum subband model, we have already obtained such estimates, which are stated in Theorem 2.5. For the 3D Schrödinger-Poisson system (1.1), (1.2), we recall the following result which was obtained in [6] (see Proposition 3.2 in this reference): 
Lemma 4.1 ([6]) Under Assumptions 2.2 and 2.6, the 3D Schrödinger-Poisson problem (1.1), (1.2) admits a unique global solution, which satisfies

$$
\begin{gathered}
\forall s \in[2, \infty) \quad\left\|\psi^{3 \mathrm{D}}\right\|_{L_{t}^{s^{*}} W_{x}^{1, s} L_{z}^{2}} \leq C, \\
\left\|V^{3 \mathbf{D}}\right\|_{L_{t, x, z}^{\infty}} \leq C,
\end{gathered}
$$

where $s^{*}$ is defined in (2.1) and $C$ is independent of $\varepsilon$.

Before proving Theorem 2.7, let us improve the estimates of the quantum subband model in the case of strong solutions.

Lemma 4.2 Let $q \in(2, \infty)$. Under Assumptions 2.2, 2.3, 2.6, if $\varepsilon$ is small enough, let $\left(\phi_{p}^{\mathrm{SB}}, V^{\mathrm{SB}}\right)$ be the solution of (1.4), (1.5), (1.6). Then there exists a constant $C>0$ independent of $\varepsilon$ such that

$$
\left\|\nabla_{x, z} \Delta_{x} V^{\mathrm{SB}}\right\|_{L_{t}^{\infty} L_{x}^{q} L_{z}^{\infty}}+\left\|\nabla_{x, z} \partial_{t} V^{\mathrm{SB}}\right\|_{L_{t}^{\infty} L_{x}^{q} L_{z}^{\infty}} \leq C .
$$

Proof. Denote $u=e^{i E_{p} t / \varepsilon^{2}} \nabla_{x} \phi_{p}^{\mathbf{S B}}$. By differentiating (1.4), we get

$$
i \partial_{t} u=-\frac{1}{2} \Delta_{x} u+\left(\epsilon_{p}^{\mathrm{SB}}-\frac{E_{p}}{\varepsilon^{2}}\right) u+\phi_{p}^{\mathrm{SB}} e^{i E_{p} t / \varepsilon^{2}} \nabla_{x} \epsilon_{p}^{\mathrm{SB}} .
$$

By (2.8), (2.10) and the Sobolev embedding $W^{1, q}\left(\mathbb{R}^{2}\right) \subset L^{\infty}\left(\mathbb{R}^{2}\right)$ (recall that $q>2$ ), we have

$$
\left\|\left(\epsilon_{p}^{\mathrm{SB}}-\frac{E_{p}}{\varepsilon^{2}}\right) u+\phi_{p}^{\mathrm{SB}} e^{i E_{p} t / \varepsilon^{2}} \nabla_{x} \epsilon_{p}^{\mathrm{SB}}\right\|_{L_{t}^{1} L_{x}^{2}} \leq C .
$$

Therefore a Strichartz estimate [13] in dimension 2 implies, for all $s \in[2, \infty)$,

$$
\left\|\phi_{p}^{\mathrm{sB}}\right\|_{L_{t}^{s^{*}} W_{x}^{1, s}} \leq C .
$$

We set now $w=\Delta_{x} \phi_{p}^{\text {SB }}$. By applying the Laplace operator to (1.4), we get

$$
i \partial_{t} w=-\frac{1}{2} \Delta_{x} w+\epsilon_{p}^{\mathrm{sB}} w+S_{1}+S_{2}
$$

where the source terms in this Schrödinger equation are written

$$
S_{1}=2 \nabla_{x} \epsilon_{p}^{\mathrm{SB}} \cdot \nabla_{x} \phi_{p}^{\mathrm{SB}}, \quad S_{2}=\phi_{p}^{\mathrm{SB}} \Delta_{x} \epsilon_{p}^{\mathrm{SB}}
$$

The first term is easy to estimate thanks to (2.10) and (4.4):

$$
\left\|S_{1}\right\|_{L_{t}^{1} L_{x}^{2}} \leq C\left\|\nabla_{x} \epsilon_{p}^{\mathrm{SB}}\right\|_{L_{t}^{\infty} L_{x}^{q}}\left\|\nabla_{x} \phi_{p}^{\mathrm{SB}}\right\|_{L_{t}^{q} L_{x}^{q^{*}}} \leq C .
$$

Let us now estimate $S_{2}(t)$, pointwise in time. Straightforward computations lead to

$$
\Delta_{x} \epsilon_{p}^{\mathrm{SB}}=\left\langle\left|\chi_{p}^{\mathrm{SB}}\right|^{2} \Delta_{x} V^{\mathrm{SB}}\right\rangle+\sum_{k \neq p} \frac{\left\langle\chi_{p}^{\mathrm{SB}} \chi_{k}^{\mathrm{SB}} \nabla_{x} V^{\mathrm{SB}}\right\rangle^{2}}{\epsilon_{p}^{\mathrm{SB}}-\epsilon_{k}^{\mathrm{SB}}}
$$


As it has been shown in Section 3.1, for $\varepsilon<\varepsilon(M, q)$ we have

$$
\forall k \neq p \quad\left|\epsilon_{p}^{\mathrm{SB}}-\epsilon_{k}^{\mathrm{SB}}\right| \geq \frac{C}{\varepsilon^{2}} .
$$

Consequently, the second term in the right-hand side of (4.7) can be bounded as follows, pointwise in $t, x$ :

$$
\left|\sum_{k \neq p} \frac{\left\langle\chi_{p}^{\mathrm{SB}} \chi_{k}^{\mathrm{SB}} \nabla_{x} V^{\mathrm{SB}}\right\rangle^{2}}{\epsilon_{p}^{\mathrm{SB}}-\epsilon_{k}^{\mathrm{SB}}}\right| \leq C \varepsilon^{2} \sum_{k \neq p}\left\langle\chi_{p}^{\mathrm{SB}} \chi_{k}^{\mathrm{SB}} \nabla_{x} V^{\mathrm{SB}}\right\rangle^{2} \leq C \varepsilon^{2}\left\|\nabla_{x} V^{\mathrm{SB}}\right\|_{L_{z}^{\infty}}^{2} .
$$

Furthermore, by using (2.9), (2.11) with $2 q$ (up to another modification of $\varepsilon(M, q)$ ), we get

$$
\left\|\nabla_{x, z} V^{\mathrm{SB}}\right\|_{L_{t}^{\infty} L_{x}^{2 q} L_{z}^{\infty}}+\left\|\nabla_{x} \chi_{p}^{\mathrm{SB}}\right\|_{L_{t}^{\infty} L_{x}^{2 q} L_{z}^{2}} \leq C,
$$

thus

$$
\left\|\sum_{k \neq p} \frac{\left\langle\chi_{p}^{\mathrm{SB}} \chi_{k}^{\mathrm{SB}} \nabla_{x} V^{\mathrm{SB}}\right\rangle^{2}}{\epsilon_{p}^{\mathrm{SB}}-\epsilon_{k}^{\mathrm{SB}}}\right\|_{L_{t}^{\infty} L_{x}^{q}} \leq C \varepsilon^{2}\left\|\nabla_{x} V^{\mathrm{SB}}\right\|_{L_{t}^{\infty} L_{x}^{2 q} L_{z}^{\infty}}^{2} \leq C \varepsilon^{2} .
$$

The first term in the right-hand side of (4.7) is more delicate to estimate and requires an argument similar as the one used previously in Step 3 of the proof of Proposition 3.2. We only sketch this argument and leave the details to the reader. First, by computing straightforwardly $\Delta_{x} \chi_{p}^{\mathrm{sB}}$, we obtain

$$
\begin{aligned}
\left\|\Delta_{x} \chi_{p}^{\mathrm{SB}}(t)\right\|_{L_{x}^{q} L_{z}^{2}} & \leq C \varepsilon^{4}\left\|\nabla_{x} V^{\mathrm{SB}}\right\|_{L_{t}^{\infty} L_{x}^{2 q} L_{z}^{\infty}}^{2}+C \varepsilon^{2}\left\|\Delta_{x} V^{\mathrm{SB}}(t)\right\|_{L_{x}^{q} L_{z}^{\infty}} \\
& \leq C \varepsilon^{2}\left(1+\left\|\Delta_{x} V^{\mathrm{SB}}(t)\right\|_{L_{x}^{q} L_{z}^{\infty}}\right)
\end{aligned}
$$

where we used (4.9). If the Laplace operator is applied to the Poisson equation 1.6, it comes

$$
\Delta_{x} V^{\mathrm{SB}}=\frac{2}{4 \pi r} *\left[\left(\mathcal{R} e\left(\overline{\phi_{p}^{\mathrm{SB}}} \Delta_{x} \phi_{p}^{\mathrm{SB}}\right)+\left|\nabla_{x} \phi_{p}^{\mathrm{SB}}\right|^{2}\right)\left|\chi_{p}^{\mathrm{SB}}\right|^{2}+\left|\phi_{p}^{\mathrm{SB}}\right|^{2}\left(\chi_{p}^{\mathrm{SB}} \Delta_{x} \chi_{p}^{\mathrm{SB}}+\left|\nabla_{x} \chi_{p}^{\mathrm{SB}}\right|^{2}\right)\right] .
$$

Hölder inequalities and Sobolev embedding imply that

$$
\begin{aligned}
\left\|\left|\nabla_{x} \phi_{p}^{\mathrm{SB}}\right|^{2}(t)\right\|_{L_{x}^{q \#}} & \leq\left\|\nabla_{x} \phi_{p}^{\mathrm{SB}}(t)\right\|_{L_{x}^{2}}\left\|\nabla_{x} \phi_{p}^{\mathrm{SB}}(t)\right\|_{L_{x}^{q}} \\
& \leq C\left\|\nabla_{x} \phi_{p}^{\mathrm{SB}}\right\|_{L_{t}^{\infty} L_{x}^{2}}\left(\left\|\Delta_{x} \phi_{p}^{\mathrm{SB}}(t)\right\|_{L_{x}^{2}}+\left\|\phi_{p}^{\mathrm{SB}}\right\|_{L_{t}^{\infty} L_{x}^{2}}\right)
\end{aligned}
$$

Hence, by using (2.8), (4.9), (4.11), (2.2) (and up to another modification of $\varepsilon(M, q)$ ), we obtain

$$
\left\|\Delta_{x} V^{\mathrm{SB}}(t)\right\|_{L_{x}^{q} L_{z}^{\infty}} \leq C+C\left\|\Delta_{x} \phi_{p}^{\mathrm{SB}}(t)\right\|_{L_{x}^{2}} .
$$

Then (4.7), (4.10) and (2.8) yield

$$
\left\|S_{2}(t)\right\|_{L_{x}^{2}} \leq C+C\left\|\Delta_{x} \phi_{p}^{\mathrm{SB}}(t)\right\|_{L_{x}^{2}} .
$$


From (4.5) and (4.6), we deduce that

$$
\forall t \quad\left\|\Delta_{x} \phi_{p}^{\mathrm{SB}}(t)\right\|_{L_{x}^{2}} \leq C+C \int_{0}^{t}\left\|\Delta_{x} \phi_{p}^{\mathrm{SB}}(s)\right\|_{L_{x}^{2}} d s
$$

and a Gronwall lemma enables to conclude that

$$
\begin{gathered}
\left\|\phi_{p}^{\mathrm{SB}}\right\|_{L_{t}^{\infty} H_{x}^{2}} \leq C, \\
\left\|\Delta_{x} V^{\mathrm{SB}}\right\|_{L_{t}^{\infty} L_{x}^{q} L_{z}^{\infty}}+\frac{1}{\varepsilon^{2}}\left\|\Delta_{x} \chi_{p}^{\mathrm{SB}}\right\|_{L_{t}^{\infty} L_{x}^{q} L_{z}^{2}} \leq C,
\end{gathered}
$$

where (4.12) and (4.11) were used again.

The equation of charge conservation is written

$$
\partial_{t}\left(\left|\phi_{p}^{\mathbf{S B}}\right|^{2}\right)+\operatorname{div}_{x} 2 \mathcal{I} m\left(\overline{\phi_{p}^{\mathbf{S B}}} \nabla_{x} \phi_{p}^{\mathbf{S B}}\right)=0,
$$

thus it can be inferred from (4.13) and (2.8) that

$$
\left\|\partial_{t}\left(\left|\phi_{p}^{\mathrm{SB}}\right|^{2}\right)\right\|_{L_{t}^{\infty} L_{x}^{q}} \leq C .
$$

Since we have

$$
\partial_{t} V^{\mathrm{SB}}=\frac{1}{4 \pi r} *\left[\partial_{t}\left(\left|\phi_{p}^{\mathrm{SB}}\right|^{2}\right)\left|\chi_{p}^{\mathrm{SB}}\right|^{2}+\left|\phi_{p}^{\mathrm{SB}}\right|^{2} \partial_{t}\left(\left|\chi_{p}^{\mathrm{SB}}\right|^{2}\right)\right]
$$

we claim that, for $\varepsilon$ small enough and by following the method already used above in the proof of Proposition 3.2 (Step 3), this enables to show the estimate:

$$
\left\|\partial_{t} V^{\mathrm{SB}}\right\|_{L_{t}^{\infty} L_{x}^{q} L_{z}^{\infty}}+\frac{1}{\varepsilon^{2}}\left\|\partial_{t} \chi_{p}^{\mathrm{SB}}\right\|_{L_{t}^{\infty} L_{x}^{q} L_{z}^{2}} \leq C .
$$

Next, if $\phi_{p, 0}$ belongs to $H^{3}\left(\mathbb{R}^{2}\right)$, one can reiterate the proof by following the same strategy and estimate higher order derivatives of $V^{\mathrm{sB}}$. Let us sketch this proof. By differentiating (4.5), one gets a Schrödinger equation on $\nabla_{x} \Delta_{x} \phi_{p}^{\text {sB }}$, with several source terms. Thanks to bounds that have already been obtained, all these terms can be estimates in $L_{t}^{1} L_{x}^{2}$, except for one term, which requires more work:

$$
S_{3}=\left\langle\left|\chi_{p}^{\mathrm{SB}}\right|^{2} \nabla_{x} \Delta_{x} V^{\mathrm{SB}}\right\rangle \phi_{p}^{\mathrm{SB}}
$$

Then it suffices to show that

$$
\left\|\nabla_{x} \Delta_{x} \chi_{p}^{\mathrm{SB}}(t)\right\|_{L_{x}^{q} L_{z}^{2}} \leq C \varepsilon^{2}\left(1+\left\|\nabla_{x} \Delta_{x} V^{\mathrm{SB}}\right\|_{L_{x}^{q} L_{z}^{\infty}}\right)
$$

and use the Poisson equation (after the application of $\nabla_{x} \Delta_{x}$ to this equation) to get, for $\varepsilon$ small enough, a bound for $\nabla_{x, z} \Delta_{x} V^{\mathrm{SB}}$ in terms of $\nabla_{x} \Delta_{x} \phi_{p}^{\mathrm{SB}}$ and deduce

$$
\left\|S_{3}\right\|_{L_{t}^{1} L_{x}^{2}} \leq C+C \int_{0}^{t}\left\|\nabla_{x} \Delta_{x} \phi_{p}^{\mathrm{SB}}(s)\right\|_{L_{x}^{2}} d s .
$$


We conclude as above with a Gronwall lemma. By using also (4.15), we finally obtain (4.3).

Proof of Theorem 2.7. Let us now estimate the discrepancy between the quantum subband model and the 3D model (1.1), (1.2). We denote by $\Pi_{p}^{\mathrm{sB}}$ the projector in $L^{2}(\mathbb{R})$ on the $p$ th subband, i.e.

$$
\forall \psi \in L^{2}(\mathbb{R}) \quad \Pi_{p}^{\mathrm{SB}} \psi=\left\langle\psi \chi_{p}^{\mathrm{SB}}\right\rangle \chi_{p}^{\mathrm{SB}} .
$$

Thanks to the estimates (2.9) and (4.3), one can deduce the crucial following bounds from Lemma A.4 of the Appendix:

$$
\left\|\nabla_{x} \chi_{p}^{\mathrm{SB}}\right\|_{L_{t}^{\infty} L_{x}^{q} L_{z}^{2}}+\left\|\Delta_{x} \chi_{p}^{\mathrm{SB}}\right\|_{L_{t}^{\infty} L_{x}^{q} L_{z}^{2}}+\left\|\partial_{t} \chi_{p}^{\mathrm{SB}}\right\|_{L_{t}^{\infty} L_{x}^{q} L_{z}^{2}} \leq C \varepsilon^{3} .
$$

Then, as a direct consequence, we get a bound for the commutators between $\Pi_{p}^{\mathrm{SB}}$ and the operators of differentiation with respect to $t$ and $x$. Indeed, straightforward calculations show that for any $\psi \in L_{x}^{q^{*}} L_{z}^{2}$ we have

$$
\left\|\left[\partial_{t}, \Pi_{p}^{\mathrm{SB}}\right] \psi\right\|_{L^{2}\left(\mathbb{R}^{3}\right)}+\left\|\left[\nabla_{x}, \Pi_{p}^{\mathrm{SB}}\right] \psi\right\|_{L^{2}\left(\mathbb{R}^{3}\right)} \leq C \varepsilon^{3}\|\psi\|_{q^{*}, 2}
$$

and, if in addition we have $\nabla_{x} \psi \in L_{x}^{q^{*}} L_{z}^{2}$,

$$
\left\|\left[\Delta_{x}, \Pi_{p}^{\mathrm{SB}}\right] \psi\right\|_{L^{2}\left(\mathbb{R}^{3}\right)} \leq C \varepsilon^{3}\|\psi\|_{W_{x}^{1, q^{*}}\left(L_{z}^{2}\right)} .
$$

Of course, the same estimates hold for $\mathbb{Q}_{p}^{\mathrm{SB}}:=\mathbb{I}-\Pi_{p}^{\mathrm{SB}}$. Furthermore, it is also readily seen that the operator

$$
\psi \in L^{2}\left(\mathbb{R}^{3}\right) \longmapsto \mathbb{P}_{p}^{\mathrm{SB}}(\psi):=\left\langle\phi \chi_{p}^{\mathrm{SB}}\right\rangle \in L^{2}\left(\mathbb{R}^{2}\right)
$$

also satisfies the same kind of commutator estimates: if $\psi \in W_{x}^{1, q^{*}}\left(L_{z}^{2}\right)$ then

$$
\begin{gathered}
\left\|\left[\partial_{t}, \mathbb{P}_{p}^{\mathrm{SB}}\right] \psi\right\|_{L^{2}\left(\mathbb{R}^{2}\right)}+\left\|\left[\nabla_{x}, \mathbb{P}_{p}^{\mathrm{SB}}\right] \psi\right\|_{L^{2}\left(\mathbb{R}^{2}\right)} \leq C \varepsilon^{3}\|\psi\|_{q^{*}, 2}, \\
\left\|\left[\Delta_{x}, \mathbb{P}_{p}^{\mathrm{SB}}\right] \psi\right\|_{L^{2}\left(\mathbb{R}^{2}\right)} \leq C \varepsilon^{3}\|\psi\|_{W_{x}^{1, q^{*}\left(L_{z}^{2}\right)}} .
\end{gathered}
$$

Let us now introduce the two following operators:

$$
A^{\varepsilon}=-\frac{1}{2} \partial_{z}^{2}+V_{c}^{\varepsilon} \quad ; \quad H^{\mathrm{SB}}=A^{\varepsilon}+V^{\mathrm{SB}}=-\frac{1}{2} \partial_{z}^{2}+V_{c}^{\varepsilon}+V^{\mathrm{SB}} .
$$

The 3D Schrödinger equation (1.1) can be rewritten:

$$
i \partial_{t} \psi^{3 \mathbf{D}}=-\frac{1}{2} \Delta_{x} \psi^{3 \mathbf{D}}+H^{\mathrm{SB}} \psi^{3 \mathbf{D}}+\left(V^{3 \mathbf{D}}-V^{\mathrm{SB}}\right) \psi^{3 \mathbf{D}} .
$$

Let us decompose $\psi^{3 \mathrm{D}}$ as follows:

$$
\psi^{3 \mathrm{D}}=\psi_{p}^{3 \mathrm{D}}+\omega=\phi_{p}^{3 \mathrm{D}} \chi_{p}^{\mathrm{SB}}+\omega,
$$


with

$$
\psi_{p}^{3 \mathbf{D}}=\Pi_{p}^{\mathrm{SB}} \psi^{3 \mathrm{D}}, \quad \phi_{p}^{3 \mathbf{D}}=\left\langle\psi_{p}^{3 \mathbf{D}} \chi_{p}^{\mathrm{SB}}\right\rangle=\mathbb{P}_{p}^{\mathrm{SB}} \psi^{3 \mathrm{D}}, \quad \omega=\mathbb{Q}_{p}^{\mathrm{SB}} \psi^{3 \mathrm{D}}=\psi^{3 \mathbf{D}}-\psi_{p}^{3 \mathbf{D}} .
$$

At the initial time, by Assumption 2.6, we have

$$
\phi_{p}^{\mathbf{3 D}}=\phi_{p}^{\mathrm{SB}}\left\langle X_{p}^{\varepsilon} \chi_{p}^{\mathrm{SB}}\right\rangle, \quad \omega=\phi_{p}^{\mathrm{SB}}\left(X_{p}^{\varepsilon}-\left\langle X_{p}^{\varepsilon} \chi_{p}^{\mathrm{SB}}\right\rangle \chi_{p}^{\mathrm{SB}}\right),
$$

thus (A.8) implies that

$$
\left\|\left(\phi_{p}^{3 \mathrm{D}}-\phi_{p}^{\mathrm{SB}}\right)(0, \cdot)\right\|_{L^{2}\left(\mathbb{R}^{2}\right)}+\|\omega(0, \cdot, \cdot)\|_{L^{2}\left(\mathbb{R}^{3}\right)} \leq C \varepsilon^{3} .
$$

Since $H^{\mathrm{SB}}$ commutes with $\Pi_{p}^{\mathrm{SB}}$ and $\mathbb{Q}_{p}^{\mathrm{SB}}$, and since $\Pi_{p}^{\mathrm{SB}} H^{\mathrm{SB}}=\epsilon_{p}^{\mathrm{SB}} \Pi_{p}^{\mathrm{SB}}$, straightforward calculations lead to following two equations:

$$
\begin{gathered}
i \partial_{t} \phi_{p}^{3 \mathrm{D}}=-\frac{1}{2} \Delta_{x} \phi_{p}^{3 \mathrm{D}}+\epsilon_{p}^{\mathrm{SB}} \phi_{p}^{3 \mathrm{D}}+\Lambda_{1}, \\
i \partial_{t} \omega=-\frac{1}{2} \Delta_{x} \omega+A^{\varepsilon} \omega+V^{\mathrm{SB}} \omega+\Lambda_{2},
\end{gathered}
$$

where the remainders are written:

$$
\begin{aligned}
& \Lambda_{1}=\left(-i\left[\partial_{t}, \mathbb{P}_{p}^{\mathrm{SB}}\right]-\frac{1}{2}\left[\Delta_{x}, \mathbb{P}_{p}^{\mathrm{SB}}\right]\right) \psi^{3 \mathrm{D}}+\mathbb{P}_{p}^{\mathrm{SB}}\left(\left(V^{3 \mathrm{D}}-V^{\mathrm{SB}}\right) \psi^{3 \mathrm{D}}\right), \\
& \Lambda_{2}=\left(-i\left[\partial_{t}, \mathbb{Q}_{p}^{\mathrm{SB}}\right]-\frac{1}{2}\left[\Delta_{x}, \mathbb{Q}_{p}^{\mathrm{SB}}\right]\right) \psi^{3 \mathrm{D}}+\mathbb{Q}_{p}^{\mathrm{SB}}\left(\left(V^{3 \mathrm{D}}-V^{\mathrm{SB}}\right) \psi^{3 \mathrm{D}}\right) .
\end{aligned}
$$

Let $s \in(2, \infty)$ be fixed. By the commutator estimates (4.17), (4.18) and by (4.1), it is readily seen that

$$
\left\|\Lambda_{1}\right\|_{L_{t}^{1} L_{x}^{2}}+\left\|\Lambda_{2}\right\|_{L_{t}^{1} L_{x, z}^{2}} \leq C \varepsilon^{3}+C\left\|V^{3 \mathrm{D}}-V^{\mathrm{SB}}\right\|_{L_{t}^{1} L_{x}^{s} L_{z}^{\infty}} .
$$

Consequently, by using the fact that in (4.23) the self-adjoint operator $A^{\varepsilon}$ only acts in direction $z$, a Strichartz-type inequality can be applied in directions $x$ only (such inequalities were derived in [6] thanks to some results of [12]). From (4.21), we infer

$$
\|\omega\|_{L_{t}^{a^{*}} L_{x}^{a} L_{z}^{2}} \leq C \varepsilon^{3}+C\left\|V^{3 \mathrm{D}}-V^{\mathrm{SB}}\right\|_{L_{t}^{1} L_{x}^{s} L_{z}^{\infty}}
$$

for any $a \in[2, \infty)$, with $a^{*}=\frac{2 a}{a-2}$. Furthermore, the difference $\sigma=\phi_{p}^{\mathbf{3 D}}-\phi_{p}^{\mathbf{S B}}$ is small at $t=0$ (thanks to (4.21)) and solves the equation

$$
i \partial_{t} \sigma=-\frac{1}{2} \Delta_{x} \sigma+\frac{E_{p}}{\varepsilon^{2}} \sigma+\left(\epsilon_{p}^{\mathrm{SB}}-\frac{E_{p}}{\varepsilon^{2}}\right) \sigma+\Lambda_{1} .
$$

Therefore, (2.10) and the above estimate of $\Lambda_{1}$ imply, by a Strichartz estimate in dimension 2 , that

$$
\forall a \in[2, \infty) \quad\|\sigma\|_{L_{t}^{a^{*}} L_{x}^{a}} \leq C \varepsilon^{3}+C\left\|V^{3 \mathrm{D}}-V^{\mathrm{SB}}\right\|_{L_{t}^{1} L_{x}^{s} L_{z}^{\infty}} .
$$


Let us now estimate the difference between the densities:

$$
\begin{aligned}
n^{3 \mathrm{D}}-n^{\mathrm{SB}} & =\left|\psi^{3 \mathrm{D}}\right|^{2}-\left|\phi_{p}^{\mathrm{SB}}\right|^{2}\left|\chi_{p}^{\mathrm{SB}}\right|^{2} \\
& =\left(\sigma \overline{\phi_{p}^{\mathbf{3 D}}}+\bar{\sigma} \phi_{p}^{\mathrm{SB}}\right)\left|\chi_{p}^{\mathrm{SB}}\right|^{2}+\omega \overline{\psi^{3 \mathbf{D}}}+\bar{\omega} \phi_{p}^{\mathbf{3 D}} \chi_{p}^{\mathrm{SB}}
\end{aligned}
$$

We remark that a differentiation leads to

$$
\nabla_{x} \phi_{p}^{3 \mathrm{D}}=\left\langle\nabla_{x} \psi^{3 \mathrm{D}} \chi_{p}^{\mathrm{SB}}\right\rangle+\left\langle\psi^{3 \mathrm{D}} \nabla_{x} \chi_{p}^{\mathrm{SB}}\right\rangle
$$

thus (4.1) and (2.11) imply

$$
\left\|\nabla_{x} \phi_{p}^{3 \mathrm{D}}\right\|_{L_{t}^{\infty} L_{x}^{2}} \leq C,
$$

which means, by Sobolev embeddings, that

$$
\left\|\phi_{p}^{3 \mathrm{D}}\right\|_{L_{t}^{\infty} L_{x}^{s}}+\left\|\phi_{p}^{\mathrm{SB}}\right\|_{L_{t}^{\infty} L_{x}^{s}}+\left\|\psi^{3 \mathrm{D}}\right\|_{L_{t}^{\infty} L_{x}^{s} L_{z}^{2}} \leq C,
$$

where we used again (2.8) and (4.1). Hence, by (4.24), (4.25) with $a=2$ and a Hölder inequality, it comes

$$
\left\|\left(n^{3 \mathrm{D}}-n^{\mathrm{SB}}\right)(t)\right\|_{L_{x}^{s \#} L_{z}^{1}} \leq C \varepsilon^{3}+C\left\|V^{3 \mathrm{D}}-V^{\mathrm{SB}}\right\|_{L_{t}^{1} L_{x}^{s} L_{z}^{\infty}} .
$$

Then, together with (2.2), the Poisson equation for the two models (1.6) and (1.2) implies that

$$
\left\|\left(V^{3 \mathrm{D}}-V^{\mathrm{SB}}\right)(t)\right\|_{L_{x}^{s} L_{z}^{\infty}} \leq C \varepsilon^{3}+\int_{0}^{t}\left\|\left(V^{3 \mathrm{D}}-V^{\mathrm{SB}}\right)(\tau)\right\|_{L_{x}^{s} L_{z}^{\infty}} d \tau
$$

By a Gronwall lemma, we conclude that

$$
\left\|\left(V^{3 \mathrm{D}}-V^{\mathrm{SB}}\right)\right\|_{L_{t}^{\infty} L_{x}^{s} L_{z}^{\infty}} \leq C \varepsilon^{3} .
$$

From this estimate, it is easy to complete the proof of Theorem 2.7.

\section{Comparison with another approximate model}

The following model, called the 2.5D adiabatic model, was introduced in [6] as an approximation of the 3D Schrödinger-Poisson system (1.1), (1.2):

$$
\begin{gathered}
i \partial_{t} \phi_{p}^{2.5 \mathrm{D}}=-\frac{1}{2} \Delta_{x} \phi_{p}^{2.5 \mathrm{D}}+\left(\frac{E_{p}}{\varepsilon^{2}}+\left\langle V^{2.5 \mathrm{D}}\left|X_{p}^{\varepsilon}\right|^{2}\right\rangle\right) \phi_{p}^{2.5 \mathrm{D}}, \\
V^{2.5 \mathrm{D}}=\frac{1}{4 \pi r} *\left(\left|\phi_{p}^{2.5 \mathrm{D}}\right|^{2}\left|X_{p}^{\varepsilon}\right|^{2}\right) .
\end{gathered}
$$

Recall that $\frac{E_{p}}{\varepsilon^{2}}, X_{p}^{\varepsilon}$ were defined as the spectral elements of $-\frac{1}{2} \frac{d^{2}}{d z^{2}}+V_{c}^{\varepsilon}$. This model can be seen as another "block diagonalization" of the 3D Schrödinger-Poisson system, where the basis $\left(X_{p}^{\varepsilon}\right)_{p \in \mathbb{N}^{*}}$ has been chosen for projecting the wavefunction, instead 
of the basis of the subbands $\left(\chi_{p}^{3 \mathrm{D}}\right)_{p \in \mathbb{N}^{*}}$ (which was used for the quantum subband model, see the Introduction).

Lemma A.3 says that, if $\varepsilon$ is small, $X_{p}^{\varepsilon}$ is close to $\chi_{p}\left[V^{\mathbf{2 . 5 \mathrm { D }}}\right]$ and $\frac{E_{p}}{\varepsilon^{2}}+\left\langle V^{\mathbf{2 . 5 \mathrm { D }}}\left|X_{p}^{\varepsilon}\right|^{2}\right\rangle$ is close to $\epsilon_{p}\left[V^{2.5 \mathrm{D}}\right]$. By quantifying these estimates, one can prove that the adiabatic model is close to the quantum subband model studied in this paper:

Proposition 5.1 Under Assumptions 2.2 and 2.3, let $p \in \mathbb{N}^{*}$ be given and let $\phi_{p, 0}$ belong to $H^{1}\left(\mathbb{R}^{2}\right)$. Consider $\left(\phi_{p}^{\mathrm{SB}}, \chi_{p}^{\mathrm{SB}}, V^{\mathrm{SB}}, n^{\mathrm{SB}}\right)$ and $\left(\phi_{p}^{2.5 \mathrm{D}}, V^{2.5 \mathrm{D}}, n^{2.5 \mathrm{D}}\right)$, respectively defined as the solutions of the quantum subband model (1.4), (1.5), (1.6) and of the 2.5D adiabatic model (5.1), (5.2), both with $\phi_{p, 0}$ as Cauchy datum. Then we have, for any $q \in(2, \infty)$ and $T>\infty$,

$$
\sup _{t \in[0, T]}\left(\left\|\phi_{p}^{\mathrm{SB}}-\phi_{p}^{2.5 \mathrm{D}}\right\|_{L_{x}^{2}}+\left\|V^{\mathrm{SB}}-V^{2.5 \mathrm{D}}\right\|_{L_{x}^{q} L_{z}^{\infty}}+\left\|n^{\mathrm{SB}}-n^{2.5 \mathrm{D}}\right\|_{L_{x, z}^{1}}\right)=\mathcal{O}\left(\varepsilon^{3}\right) .
$$

Proof. In [6], we have shown that (5.1), (5.2) is well-posed and that its solution satisfies the following estimates:

$$
\left\|V^{\mathbf{2 . 5 \mathrm { D }}}\right\|_{L_{t, x, z}^{\infty}} \leq C, \quad \forall s \in[2, \infty), \quad\left\|\phi^{2.5 \mathrm{D}}\right\|_{L_{t}^{s^{*}} W_{x}^{1, s}} \leq C,
$$

where $s^{*}$ is defined in (2.1) and $C$ is independent of $\varepsilon$. Denote

$$
u=e^{i E_{p} t / \varepsilon^{2}}\left(\phi_{p}^{\mathrm{SB}}-\phi_{p}^{2.5 \mathrm{D}}\right), \quad v=V^{\mathrm{SB}}-V^{2.5 \mathrm{D}} .
$$

The function $u$ vanishes for $t=0$ and satisfies the equation

$$
i \partial_{t} u=-\frac{1}{2} \Delta_{x} u+\left\langle V^{2.5 \mathrm{D}}\left|X_{p}^{\varepsilon}\right|^{2}\right\rangle u+R_{1}+R_{2}
$$

with

$$
\begin{gathered}
R_{1}=\left(\epsilon_{p}^{\mathrm{SB}}-\frac{E_{p}}{\varepsilon^{2}}-\left\langle V^{\mathrm{SB}}\left|X_{p}^{\varepsilon}\right|^{2}\right\rangle\right) \phi_{p}^{\mathrm{SB}} \\
R_{2}=\left\langle v\left|X_{p}^{\varepsilon}\right|^{2}\right\rangle \phi_{p}^{\mathrm{SB}} .
\end{gathered}
$$

By (A.8), it is clear that

$$
\left\|R_{1}\right\|_{L_{t}^{\infty} L_{x}^{2}} \leq C \varepsilon^{3}\left\|\partial_{z} V^{\mathrm{SB}}\right\|_{L_{t}^{\infty} L_{x}^{q} L_{z}^{\infty}}\left\|\phi_{p}^{\mathrm{SB}}\right\|_{L_{t}^{\infty} L_{x}^{q^{*}}} \leq C \varepsilon^{3},
$$

where we used (2.8), (2.9) and Sobolev embeddings. In order to treat $R_{2}$, we recall that

$$
v=\frac{1}{4 \pi r} *\left[\left(\left|\phi_{p}^{\mathrm{SB}}\right|^{2}-\left|\phi_{p}^{2.5 \mathrm{D}}\right|^{2}\right)\left|X_{p}^{\varepsilon}\right|^{2}+\left|\phi_{p}^{\mathrm{SB}}\right|^{2}\left(\left|\chi_{p}\left[V^{\mathrm{SB}}\right]\right|^{2}-\left|X_{p}^{\varepsilon}\right|^{2}\right)\right] .
$$

Hence, we deduce from (2.2) and interpolation estimates that, pointwise in time,

$$
\begin{aligned}
\|v(t)\|_{q, \infty} \leq & C\|u(t)\|_{L^{2}\left(\mathbb{R}^{2}\right)}\left(\left\|\phi_{p}^{\mathrm{SB}}\right\|_{L_{t}^{\infty} L_{x}^{q}}+\left\|\phi_{p}^{2.5 \mathrm{D}}\right\|_{L_{t}^{\infty} L_{x}^{q}}\right) \\
& +\left\|\phi_{p}^{\mathrm{SB}}\right\|_{L_{t}^{\infty} L_{x}^{4}}^{2}\left\|\chi_{p}\left[V^{\mathrm{SB}}\right]-X_{p}^{\varepsilon}\right\|_{L_{t}^{\infty} L_{x}^{q} L_{z}^{2}} \\
\leq & C\|u(t)\|_{L^{2}\left(\mathbb{R}^{2}\right)}+C \varepsilon^{3}
\end{aligned}
$$


where we used (2.8), (2.9) and, again, (A.8). Next we obtain

$$
\left\|R_{2}(t)\right\|_{L^{2}\left(\mathbb{R}^{2}\right)} \leq C\left(\|u(t)\|_{L^{2}\left(\mathbb{R}^{2}\right)}+\varepsilon^{3}\right)\left\|\phi_{p}^{\mathrm{SB}}\right\|_{L_{t}^{\infty} L_{x}^{q^{*}}} \leq C\|u(t)\|_{L^{2}\left(\mathbb{R}^{2}\right)}+C \varepsilon^{3} .
$$

Finally, thanks to these estimates of $R_{1}$ and $R_{2},(5.5)$ yields

$$
\forall t \in[0, T], \quad\|u(t)\|_{L^{2}\left(\mathbb{R}^{2}\right)} \leq C \varepsilon^{3}+C \int_{0}^{t}\|u(s)\|_{L^{2}\left(\mathbb{R}^{2}\right)} d s .
$$

By a Gronwall lemma, we conclude that

$$
\|u\|_{L_{t}^{\infty} L_{x}^{2}} \leq C \varepsilon^{3}
$$

thus (5.7) yields

$$
\left\|V^{\mathrm{SB}}-V^{2.5 \mathrm{D}}\right\|_{L_{t}^{\infty} L_{x}^{q} L_{z}^{\infty}} \leq C \varepsilon^{3} .
$$

Moreover, by a Strichartz estimate [13] in dimension 2, we have for any $s \in[2, \infty)$, $s^{*}=\frac{2 q}{q-2}$,

$$
\|u\|_{L_{t}^{s^{*} L_{x}^{s}}} \leq C\left\|\left\langle V^{2.5 \mathrm{D}}\left|X_{p}^{\varepsilon}\right|^{2}\right\rangle u+R_{1}+R_{2}\right\|_{L_{t}^{1} L_{x}^{2}} \leq C \varepsilon^{3} .
$$

By using again (5.6) and (2.4), we obtain now for any $\alpha \in[1, \infty)$ :

$$
\left\|V^{\mathrm{SB}}-V^{2.5 \mathrm{D}}\right\|_{L_{t}^{\alpha} L_{x}^{\infty} L_{z}^{\infty}} \leq C \varepsilon^{3} .
$$

Remark that during this proof, we have obtained the following intermediate result, concerning the densities $n^{\mathrm{SB}}=\left|\phi_{p}^{\mathrm{SB}}\right|^{2}\left|\chi_{p}^{\mathrm{SB}}\right|^{2}$ and $n^{2.5 \mathrm{D}}=\left|\phi_{p}^{2.5 \mathrm{D}}\right|^{2}\left|X_{p}^{\varepsilon}\right|^{2}$ :

$$
\forall s \in[1,2) \quad\left\|n^{\mathrm{SB}}-n^{2.5 \mathrm{D}}\right\|_{L_{t}^{\infty} L_{x}^{s} L_{z}^{1}} \leq C \varepsilon^{3} .
$$

Remark 5.2 By combining Theorem 2.7 and this Proposition 5.1, we improve a result which was obtained in [6]. In this article (for weak solutions in energy space), the difference $V^{3 D}-V^{2.5 D}$ was proved to be of order (almost) $\mathcal{O}\left(\varepsilon^{2}\right)$. Here, we complete this result by showing that, for strong solutions in $H^{3}$, this difference is of order $\mathcal{O}\left(\varepsilon^{3}\right)$.

\section{Appendix}

\section{Spectral properties of the one-dimensional Hamil- tonian}

For any function $U \in L^{\infty}(\mathbb{R})$, under Assumption 2.2, the Hamiltonian $H[U]=$ $-\frac{1}{2} \frac{d^{2}}{d z^{2}}+V_{c}^{\varepsilon}+U$ on $L^{2}(\mathbb{R})$ with the domain

$$
D(H)=\left\{\psi \in H^{2}(\mathbb{R}): \quad V_{c}^{\varepsilon} \psi \in L^{2}(\mathbb{R})\right\}
$$


admits a discrete spectrum and a complete set of eigenfunctions (see for instance [26]). Its eigenvalues and eigenfunctions are denoted by $\epsilon_{p}[U]$ and $\chi_{p}[U]$. In this appendix, we state several properties of these spectral elements which are used in this article.

First, a classical consequence of the min-max formula is the inequality:

$$
\forall U, V \in L^{\infty}(\mathbb{R}) \quad\left|\epsilon_{p}[U]-\epsilon_{p}[V]\right| \leq\|U-V\|_{\infty} .
$$

Since the space dimension in one, the eigenfunctions $\chi_{p}[V]$ are always nondegenerate and the perturbation theory [20] can be applied. The following technical lemma states without proof two useful results:

Lemma A.1 The applications $V \mapsto \epsilon_{p}[V]$ and $V \mapsto \chi_{p}[V]$ are Gâteaux differentiable on $L^{\infty}(\mathbb{R})$. The first derivatives of these applications are respectively given by

$$
\begin{gathered}
\partial_{V} \epsilon_{p}[V] \cdot W=\left\langle\left|\chi_{p}[V]\right|^{2} W\right\rangle . \\
\partial_{V} \chi_{p}[V] \cdot W=\sum_{k \neq p} \frac{\left\langle\chi_{p}[V] \chi_{k}[V] W\right\rangle}{\epsilon_{p}[V]-\epsilon_{k}[V]} \chi_{k}[V] .
\end{gathered}
$$

Thanks to this Lemma, one can show the following results:

Lemma A.2 Let $V_{1}, V_{2}, U_{1}, U_{2}$ belong to $L^{\infty}(\mathbb{R})$ and be such that

$$
\text { for } i=1,2, \quad\left\|V_{i}\right\|_{\infty} \leq \frac{\delta_{0}}{4 \varepsilon^{2}},
$$

where $\delta_{0}$ is defined in (2.6). Then for all $p \in \mathbb{N}^{*}$, the following estimates hold:

$$
\begin{gathered}
\left\|\chi_{p}\left[V_{2}\right]-\chi_{p}\left[V_{1}\right]\right\|_{2} \leq C \varepsilon^{2}\left\|V_{2}-V_{1}\right\|_{\infty}, \\
\left\|\partial_{V} \chi_{p}\left[V_{1}\right] \cdot U_{1}-\partial_{V} \chi_{p}\left[V_{2}\right] \cdot U_{2}\right\|_{2} \leq C \varepsilon^{4}\left\|V_{1}-V_{2}\right\|_{\infty}\left(\left\|U_{1}\right\|_{\infty}+\left\|U_{2}\right\|_{\infty}\right) \\
+C \varepsilon^{2}\left\|U_{1}-U_{2}\right\|_{\infty} .
\end{gathered}
$$

In these estimates, $C$ is a constant independent of $\varepsilon$ and $p$.

Proof. Introduce the following two functions of the variable $\lambda: \chi_{p}(\lambda):=\chi_{p}[(1-$ $\left.\lambda) V_{1}+\lambda V_{2}\right]$ and $\epsilon_{p}(\lambda):=\epsilon_{p}\left[(1-\lambda) V_{1}+\lambda V_{2}\right]$ for $\lambda \in[0,1]$. Lemma A.1 shows that these functions are (at least) $C^{1}$ and that

$$
\frac{d}{d \lambda} \chi_{p}(\lambda)=\sum_{k \neq p} \frac{\left\langle\chi_{p}(\lambda) \chi_{k}(\lambda)\left(V_{2}-V_{1}\right)\right\rangle}{\epsilon_{p}(\lambda)-\epsilon_{k}(\lambda)} \chi_{k}(\lambda) .
$$

Hence

$$
\left\|\frac{d}{d \lambda} \chi_{p}(\lambda)\right\|_{2}^{2}=\sum_{k \neq p} \frac{\left\langle\chi_{p}(\lambda) \chi_{k}(\lambda)\left(V_{2}-V_{1}\right)\right\rangle^{2}}{\left(\epsilon_{p}(\lambda)-\epsilon_{k}(\lambda)\right)^{2}} .
$$


Recall that $\delta_{0}>0$, thanks to Assumption 2.3. Then, by (A.2) and (A.1), we have

$$
\forall p \quad\left|\epsilon_{p}(\lambda)-E_{p} / \varepsilon^{2}\right| \leq\left\|(1-\lambda) V_{1}+\lambda V_{2}\right\|_{\infty} \leq \frac{\delta_{0}}{4 \varepsilon^{2}} .
$$

Hence we deduce

$$
\left|\epsilon_{p}(\lambda)-\epsilon_{k}(\lambda)\right| \geq\left|E_{p} / \varepsilon^{2}-E_{k} / \varepsilon^{2}\right|-\left|\epsilon_{p}(\lambda)-E_{p} / \varepsilon^{2}\right|-\left|\epsilon_{k}(\lambda)-E_{k} / \varepsilon^{2}\right| \geq \frac{\delta_{0}}{2 \varepsilon^{2}}
$$

and

$$
\begin{aligned}
\left\|\frac{d}{d \lambda} \chi_{p}(\lambda)\right\|_{2} & \leq \frac{2 \varepsilon^{2}}{\delta_{0}}\left(\sum_{k \neq p}\left\langle\chi_{p}(\lambda) \chi_{k}(\lambda)\left(V_{1}-V_{2}\right)\right\rangle^{2}\right)^{1 / 2} \\
& \leq \frac{2 \varepsilon^{2}}{\delta_{0}}\left\|\chi_{p}(\lambda)\left(V_{1}-V_{2}\right)\right\|_{2} \leq \frac{2 \varepsilon^{2}}{\delta_{0}}\left\|V_{1}-V_{2}\right\|_{\infty},
\end{aligned}
$$

where we used the fact that $\left(\chi_{k}(\lambda)\right)_{k \geq 1}$ is an orthonormal basis of $L^{2}(\mathbb{R})$. Then (A.3) stems from

$$
\chi_{p}\left[V_{1}\right]-\chi_{p}\left[V_{2}\right]=\int_{0}^{1} \frac{d}{d \lambda} \chi_{p}(\lambda) d \lambda .
$$

In order to prove (A.4), we denote $U(\lambda)=(1-\lambda) U_{1}+\lambda U_{2}$ and $\zeta(\lambda)=\partial_{V} \chi_{p}[(1-$ $\left.\lambda) V_{1}+\lambda V_{2}\right] \cdot U(\lambda)$. Proving (A.4) amounts to estimate $\|\zeta(1)-\zeta(0)\|_{2}$. Straightforward calculations show that

$$
\begin{aligned}
\frac{d}{d \lambda} \zeta(\lambda)= & \sum_{k \neq p} \frac{\left\langle\chi_{p}^{\prime} \chi_{k} U\right\rangle}{\epsilon_{p}-\epsilon_{k}} \chi_{k}+\sum_{k \neq p} \frac{\left\langle\chi_{p} \chi_{k}^{\prime} U\right\rangle}{\epsilon_{p}-\epsilon_{k}} \chi_{k}+\sum_{k \neq p} \frac{\left\langle\chi_{p} \chi_{k} U\right\rangle}{\epsilon_{p}-\epsilon_{k}} \chi_{k}^{\prime} \\
& -\sum_{k \neq p} \frac{\left\langle\chi_{p} \chi_{k} U\right\rangle\left\langle\left(V_{1}-V_{2}\right)\left(\left|\chi_{p}\right|^{2}-\left|\chi_{k}\right|^{2}\right)\right\rangle}{\left(\epsilon_{p}-\epsilon_{k}\right)^{2}} \chi_{k}+\sum_{k \neq p} \frac{\left\langle\chi_{p} \chi_{k}\left(U_{2}-U_{1}\right)\right\rangle}{\epsilon_{p}-\epsilon_{k}} \chi_{k}
\end{aligned}
$$

(where the prime denotes the derivative with respect to $\lambda$ ). By using (A.5), (A.6), the Cauchy-Schwarz inequality and (2.7), one can estimate in $L^{2}$ the terms of the right-hand side and finally get (A.4).

For smoother potentials, one can improve the estimate (A.3) of the previous lemma:

Lemma A.3 Let $V$ belong to $W^{1, \infty}(\mathbb{R})$, such that

$$
\|V\|_{\infty} \leq \frac{\delta_{0}}{4 \varepsilon^{2}}
$$

where $\delta_{0}$ is defined in (2.6). Then for all $p \in \mathbb{N}^{*}$, the following estimate holds:

$$
\left\|\chi_{p}[V]-X_{p}^{\varepsilon}\right\|_{2}+\left|\epsilon_{p}[V]-\frac{E_{p}}{\varepsilon^{2}}-\left\langle V\left|X_{p}^{\varepsilon}\right|^{2}\right\rangle\right| \leq C_{p} \varepsilon^{3}\left\|\partial_{z} V\right\|_{\infty},
$$

where $C$ is a constant independent of $\varepsilon$ (but depending on $p$ and $\|V\|_{\infty}$ ). 
Proof. As in the proof of Lemma A.2, we consider the two functions of the variable $\lambda \in[0,1]: \chi_{p}(\lambda):=\chi_{p}[\lambda V]$ and $\epsilon_{p}(\lambda):=\epsilon_{p}[\lambda V]$. From $\left|\epsilon_{p}(\lambda)-\epsilon_{k}(\lambda)\right| \geq\left|E_{p} / \varepsilon^{2}-E_{k} / \varepsilon^{2}\right|-\left|\epsilon_{p}(\lambda)-E_{k} / \varepsilon^{2}\right|-\left|\epsilon_{k}(\lambda)-E_{k} / \varepsilon^{2}\right| \geq \frac{1}{2}\left|E_{p} / \varepsilon^{2}-E_{k} / \varepsilon^{2}\right|$, we deduce that

$$
\left\|\frac{d}{d \lambda} \chi_{p}(\lambda)\right\|_{2} \leq 2 \varepsilon^{2}\left(\sum_{k \neq p} \frac{\left\langle\chi_{p}(\lambda) \chi_{k}(\lambda) V\right\rangle^{2}}{\left(E_{p}-E_{k}\right)^{2}}\right)^{1 / 2} .
$$

To estimate this quantity, one can take advantage of the regularity of $V$ in the direction $z$ and of the orthogonality between the eigenfunctions $\chi_{k}$. Pointwise in $x$ and if $k \neq p$, we have

$$
\left|\left\langle\chi_{p} \chi_{k} V\right\rangle\right|=\left|\left\langle\chi_{p} \chi_{k}\left(V-\left.V\right|_{z=0}\right)\right\rangle\right| \leq\left\langle\left|\chi_{p}\right|\left|\chi_{k}\right||z|\left\|\partial_{z} V\right\|_{\infty}\right\rangle \leq\left\|z \chi_{p}\right\|_{2}\left\|\partial_{z} V\right\|_{\infty} .
$$

By a well-known result [26], the function $\chi_{p}$ is exponentially decreasing for $z \rightarrow \infty$. Hence $z \chi_{p}$ belongs to $L^{2}(\mathbb{R})$. Recall that one can write $\chi_{p}=\frac{1}{\sqrt{\varepsilon}} \chi_{p}^{1}\left(\frac{z}{\varepsilon}\right)$, where $\chi_{p}^{1}$ is eigenfunction of $-\frac{1}{2} \frac{d^{2}}{d z^{2}}+V_{c}(z)+\varepsilon^{2} \lambda V(\varepsilon z)$. This implies that

$$
\left\|z \chi_{p}\right\|_{2}=\varepsilon\left\|z \chi_{p}^{1}\right\|_{2} \leq C_{p} \varepsilon
$$

where $C_{p}$ depends on $p$ and $\|V\|_{\infty}$ but not on $\varepsilon$. Therefore

$$
\left\|\frac{d}{d \lambda} \chi_{p}(\lambda)\right\|_{2} \leq C_{p} \varepsilon^{3}\left\|\partial_{z} V\right\|_{\infty}\left(\sum_{q \neq p} \frac{1}{\left(E_{p}-E_{q}\right)^{2}}\right)^{1 / 2} \leq C_{p}^{\prime} \varepsilon^{3}\left\|\partial_{z} V\right\|_{\infty},
$$

where we used (2.7). Then the first part of (A.8) is a consequence of

$$
\chi_{p}[V]-X_{p}^{\varepsilon}=\int_{0}^{1} \frac{d}{d \lambda} \chi_{p}(\lambda) d \lambda .
$$

To get the estimate of $\epsilon_{p}[V]$ in (A.8), it suffices to remark that

$$
\frac{d}{d \lambda} \epsilon_{p}(\lambda)=\left\langle V\left|\chi_{p}(\lambda)\right|^{2}\right\rangle
$$

then to write

$$
\begin{aligned}
\left|\epsilon_{p}(1)-\frac{E_{p}}{\varepsilon^{2}}-\frac{d}{d \lambda} \epsilon_{p}(0)\right| & =\left|\int_{0}^{1}\left(\frac{d}{d \lambda} \epsilon_{p}(\lambda)-\frac{d}{d \lambda} \epsilon_{p}(0)\right) d \lambda\right| \\
& =\left|\int_{0}^{1}\left\langle V\left(\chi_{p}(\lambda)-X_{p}^{\varepsilon}\right)\left(\chi_{p}(\lambda)+X_{p}^{\varepsilon}\right)\right\rangle d \lambda\right| \\
& \leq 2\|V\|_{\infty} \int_{0}^{1}\left\|\chi_{p}(\lambda)-X_{p}^{\varepsilon}\right\|_{2} d \lambda \leq 2 C_{p} \varepsilon^{3}\left\|\partial_{z} V\right\|_{L_{z}^{\infty}} .
\end{aligned}
$$

Let us now consider potentials which depend on $z$ and on another variable $\lambda$ (typically in this paper, we apply these results with $\lambda=x$ or $\lambda=t$ ). By proceeding as in the proof of Lemma A.3, one can obtain the following results (the proof is left to the reader): 
Lemma A.4 Let $V=V(\lambda, z)$ belong to $L^{\infty}(\lambda, z)$, such that $\partial_{\lambda z}^{2} V \in L_{\text {loc }}^{2}\left(\lambda, L_{z}^{\infty}(\mathbb{R})\right)$ and

$$
\|V\|_{\infty} \leq \frac{\delta_{0}}{4 \varepsilon^{2}}
$$

where $\delta_{0}$ is defined in (2.6). Then for all $p \in \mathbb{N}^{*}$, the following estimate, pointwise in $\lambda$, holds:

$$
\left\|\partial_{\lambda} \chi_{p}[V]\right\|_{L_{z}^{2}} \leq C \varepsilon^{3}\left\|\partial_{\lambda z}^{2} V\right\|_{L_{z}^{\infty}}
$$

If in addition we have $\partial_{\lambda \lambda z}^{3} V \in L_{\text {loc }}^{2}\left(\lambda, L_{z}^{\infty}(\mathbb{R})\right)$, then the following estimate, pointwise in $\lambda$, holds:

$$
\left\|\partial_{\lambda \lambda}^{2} \chi_{p}[V]\right\|_{L_{z}^{2}} \leq C \varepsilon^{3}\left\|\partial_{\lambda \lambda z}^{3} V\right\|_{L_{z}^{\infty}}+C \varepsilon^{5}\left\|\partial_{\lambda z}^{2} V\right\|_{L_{z}^{\infty}}^{2} .
$$

In this Lemma, $C$ denotes a constant independent of $\varepsilon$ and $\lambda$ (but depending on $p$ and $\left.\|V\|_{\infty}\right)$.

Acknowledgement. The author acknowledges support by the European IHP network no. RNT2 2001349 entitled "Hyperbolic and kinetic equation : asymptotics, numerics, applications", by the ACI Nouvelles Interfaces des Mathématiques no. ACINIM 176-2004 entitled "MOQUA" and funded by the French ministry of research as well as the ACI Jeunes chercheurs no. JC1035 "Modèles dispersifs vectoriels pour le transport à l'échelle nanométrique". The author thanks N. Ben Abdallah for many fruitful discussions.

\section{References}

[1] T. Ando, B. Fowler, F. Stern, Electronic properties of two-dimensional systems, Rev. Mod. Phys. 54 (1982), no. 2, 437-672.

[2] A. Arnold, Self-consistent relaxation-time models in quantum mechanics, Comm. Partial Differential Equations 21 (1996), no. 3-4, 473-506.

[3] G. Bastard, Wave mechanics applied to semiconductor heterostructures, Les éditions de physique (1996).

[4] N. Ben Abdallah, F. Méhats, On a Vlasov-Schrödinger-Poisson model, Comm. Partial Differential Equations 29 (2004), no. 1-2, 173-206.

[5] N. Ben Abdallah, F. Méhats, Semiclassical analysis of the Schrödinger equation with a partially confining potential, to appear in J. Math. Pures App.

[6] N. Ben Abdallah, F. Méhats, O. Pinaud, Adiabatic approximation of the Schrödinger-Poisson system with a partial confinement, to appear in SIAM J. Math. Anal.

[7] N. Ben Abdallah, F. Méhats, G. Quinio, Global existence of classical solutions for a Vlasov-Schrödinger-Poisson system, submitted for publication. 
[8] N. Ben Abdallah, F. Méhats, N. Vauchelet, Analysis of a Drift-DiffusionSchrödinger-Poisson model, C. R. Math. Acad. Sci. Paris 2002, 335 (12), $1007-$ 1012.

[9] N. Ben Abdallah, E. Polizzi, Self-consistent three-dimensional models for quantum ballistic transport in open systems, Phys. Rev. B 66 (2002), 245301.

[10] N. Ben Abdallah, E. Polizzi, Subband decomposition approach for the simulation of quantum electron transport in nanostructures, to appear in J. Comp. Phys.

[11] F. Brezzi, P. A. Markowich, The three dimensional Wigner-Poisson Problem : existence, uniqueness and approximation, Math. Methods Appl. Sci. 14 (1991), no. $1,35-61$.

[12] F. Castella, $L^{2}$ solutions to the Schrödinger-Poisson system: existence, uniqueness, time behaviour, and smoothing effects, Math. Models Methods Appl. Sci. 7 (1997), no. 8, 1051-1083.

[13] T. Cazenave, An Introduction of Nonlinear Schrödinger Equations, Universidade Federal do Rio de Janeiro, 1996, third edition.

[14] J. H. Davies, The physics of low dimensional semiconductors, Cambridge Univ. press (1998).

[15] Yu. V. Egorov, M. A. Shubin, Linear partial differential equations. Foundations of the classical theory, Partial differential equations I, 1-259, Encyclopaedia Math. Sci., 30, Springer, Berlin, 1992.

[16] D. K. Ferry, S. M. Goodnick, Transport in nanostructures, Cambridge Univ. Press (1997).

[17] R. Froese, I. Herbst, Realizing Holonomic Constraints in Classical and Quantum Mechanics, Comm. Math. Phys. 220 (2001), no. 3, 489-535.

[18] J. Ginibre, G. Velo, The global Cauchy problem for the nonlinear Schrödinger equation revisited, Ann. Inst. H. Poincaré, Analyse non linéaire 2 (1985), no. 4, 309-327.

[19] R. Illner, P. F. Zweifel, H. Lange, Global Existence, Uniqueness and Asymptotic Behaviour of Solutions of the Wigner-Poisson and Schrödinger-Poisson Systems, Math. Methods Appl. Sci. 17 (1994), no. 5, 349-376.

[20] T. Kato, Perturbation Theory for Linear Operators, Springer-Verlag, Berlin, Heidelberg, 1966.

[21] F. Nier, A Stationary Schrödinger-Poisson System Arising from the Modelling of Electronic Devices, Forum Math. 2 (1990), no. 5, 489-510. 
[22] F. Nier, A variational formulation of Schrödinger-Poisson systems in dimension $d \leq 3$, Commun. in Partial Differential Equations 18 (1993), no. 7-8, 1125-1147.

[23] F. Nier, Schrödinger-Poisson Systems in Dimension $d \leq 3$ : The Whole-Space Case, Proc. Roy. Soc. Edinburgh Sect. A 123 (1993), no. 6, 1179-1201.

[24] A. Pazy, Semigroups of linear operators and applications to partial differential equations, Applied Mathematical Sciences, 44. Springer-Verlag, New York, 1983.

[25] O. Pinaud, Adiabatic approximation of the Schrdinger-Poisson system with a partial confinement: the stationary case, J. Math. Phys. 45 (2004), no. 5, 20292050 .

[26] M. Reed, B. Simon, Methods of Modern Mathematical Physics, Academic Press, New York, San Francisco and London, 1975.

[27] E. Polizzi, Modélisation et simulation numériques du transport quantique balistic dans les nanostructures semi-conductrices, PhD Thesis, INA Toulouse, 2001.

[28] R. S. Strichartz, Restriction of Fourier transform to quadratic surfaces and decay of solutions of wave equations, Duke Math. J. 44 (1977), no. 3, 705-714.

[29] S. Teufel, Adiabatic Perturbation Theory in Quantum Dynamics, Lecture Notes in Mathematics 1821, Springer-Verlag, Berlin, Heidelberg, New York, 2003.

[30] R. Venugopal, Z. Ren, S. Datta, M. S. Lundstrom, Simulating quantum transport in nanoscale transistors: Real versus mode-space approaches, J. Appl. Phys. 92 (2002), no. 7, 3730-3729.

[31] R. Venugopal, M. Paulsson, S. Goasgen, S. Datta, M. S. Lundstrom, A simple quantum mechanical treatment of scattering in nanoscale transistors, J. Appl. Phys. 93 (2003), no. 9, 5613-5625.

[32] J. Wang, E. Polizzi, M. S. Lundstrom, A Three-Dimensional Quantum Simulation of Silicol Nanowire Transistors with the Effective-Mass Approximation, preprint.

[33] G. Wolansky, On nonlinear stability of polytropic galaxies, Ann. Inst. Henri Poincaré 16 (1999), 15-48.

[34] K. Yajima, Existence of solutions for Schrödinger Evolution Equations, Comm. Math. Phys. 110 (1987), no. 3, 415-426. 